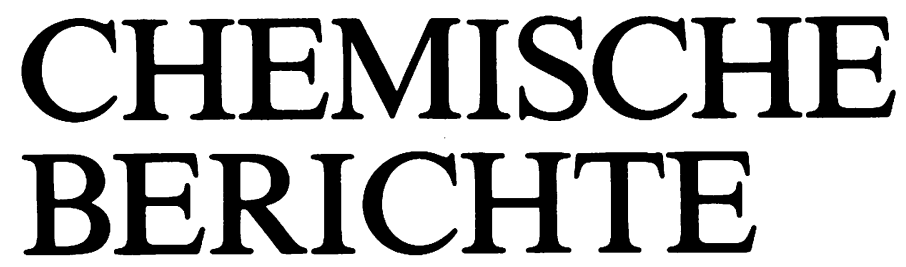

GEGRÜNDET 1868

117. JAHRGANG

HERAUSGEGEBEN IM AUFTRAG DER

GESELLSCHAFT DEUTSCHER CHEMIKER

VON

K. HAFNER - W. KIRMSE - H. MUSSO - H. NÖTH •

J. SAUER - E. WINTERFELDT

UNTER MITWIRKUNG VON

H. A. BRUNE - W. LÜTTKE - G. SPITELLER

REDAKTION: H. ZAHN

mit H. SCHILL, J. STREHLOW und A. WIELAND 


\section{BEMERKUNGEN DER REDAKTION}

1. Die Chemischen Berichte (zu zitieren als Chem. Ber.) setzen die Berichte der Deutschen Chemischen Gesellschaft (zu zitieren als Ber. Dtsch. Chem. Ges.) fort.

2. Die "Berichte“ enthalten Originalmitteilungen aus allen Gebieten der Chemie.

3. Die „Berichte“ erscheinen monatlich; ein Registerheft beschließt jeden Jahrgang.

4. Die Verantwortung für ihre Mitteilungen tragen die Verfasser selbst. - Der Korrespondenzautor ist durch einen hochgestellten Stern hervorgehoben. Die Zugehörigkeit der Autoren zu den Instituten ist durch hochgestellte Kleinbuchstaben markiert.

5. Es werden grundsätzlich nur Arbeiten aufgenommen, die vorher weder im Inland noch im Ausland veröffentlicht worden sind.

6. Eine Anweisung zur Abfassung von Manuskripten für die „Berichte“ wird auf Wunsch zugestellt.

7. Manuskripte sind zu senden an Redaktion der Chemischen Berichte. Dr. H. Zahn, Am Klopferspitz, D-8033 Martinsried, Telefon (089) 85783830.

8. Der Eingang der Abhandlungen wird den Autoren am Tage der Registrierung angezeigt.

9. Es werden nur Manuskripte in deutscher oder englischer Sprache aufgenommen. Allen Beiträgen ist eine knappe Zusammenfassung (summary) in beiden Sprachen voranzustellen, einschließlich Titel in der jeweils zweiten Sprache.

10. Der Autor muß das alleinige Urheberrecht besitzen. Mit der Annahme des Manuskriptes durch die Redaktion überträgt er dem Verlag Chemie das ausschließliche Nutzungsrecht, insbesondere das Recht der Vervielfältigung wie Fotokopie, Mikrofilm - oder mit irgendeinem anderen. Verfahren - oder das Manuskript in eine von Maschinen, insbesondere von Datenverarbeitungsmaschinen, verwendbare Sprache zu übergeben oder zu übersetzen (auch in fremde Sprachen).

11. Den Autoren werden 75 Sonderdrucke unentgeltlich portofrei zugesandt. Wünscht ein Autor mehr als 75 Abzüge, so ist dies auf dem Manuskript oder spätestens bei Rücksendung der Korrektur auf dieser zu vermerken. Den Autoren werden nur die Selbstkosten für die Zahl der die Freiexemplare überschreitenden Sonderabzüge berechnet.

12. Anfragen nach dem Verbleib nicht eingetroffener Berichte-Hefte oder Sonderdrucke sind zu richten an: Verlag Chemie GmbH, Pappelallee 3, Postfach 1260/1280, D-6940 Weinheim, Telefon (06201) 602-0.

\footnotetext{
Die Chemischen Berichte erscheinen monatlich. Der Jahresbezugspreis beträgt 770. - DM zuzügl. Versandgebühren. Einzelheft 85. - DM. In diesem Preis ist die Mehrwertsteuer enthalten. Die Bezugsbedingungen für Mitglieder der Gesellschaft Deutscher Chemiker werden auf Anfrage von der Geschäftsstelle, Carl-Bosch-Haus, Varrentrappstraße 40-42, Postfach 900440, D-6000 Frankfurt 90, mitgeteilt. - Bestellungen richten Sie bitte an Ihre Fachbuchhandlung oder unmittelbar an den Verlag. - Abbestellungen nur bis spätestens 3 Monate vor Ablauf des Kalenderjahres. - Die Lieferung erfolgt auf Rechnung und Gefahr des Empfängers. Gerichtsstand und Erfüllungsort: Weinheim/Bergstr.

Verlag und Anzeigenabteilung: Verlag Chemie GmbH, Postfach 1260/1280, D-6940 Weinheim. Telefon (06201) 602-0, Telex 465516 vchwh d, Telefax (06201) 602328.

For U.S.A., Canada, Mexico, Central and South America:

Published monthly by Verlag Chemie GmbH, Weinheim, Federal Republic of Germany, Annual subscription price: $\$ 505.00$ including postage. Second-class postage paid at Jamaica, NY 11431 . Printed in the Federal Republic of Germany. Air-freighted and mailed in the United States by Publications Expediting Inc., 200 Meacham Avenue, Elmont, NY 11003. Subscribers should place their orders through Verlag Chemie International Inc., $303 \mathrm{~N}$.W. 12 th Avenue, Deerfield Beach, Florida 33441, U.S.A.
}

U.S. Postmasters: Send address changes for Chemische Berichte to Verlag Chemie International Inc., 303 N.W. 12th Avenue, Deerfield Beach, FL 33441. 
117. Jahrgang 1984

\section{ANORGANISCHE CHEMIE}

Herrmann Wolfgang A., Bauer Christine und Weichmann Josef: ÜbergangsmetallMethylen-Komplexe, XLIII: Drei- und viergliedrige Dimetallacyclen mit Schwefeldioxid, Selen, Chlorogold und einem Silberdiazoalkan als Molekülbausteinen ......

Brunner Henri, Reiter Barbara und Riepl Georg: Asymmetrische Katalysen, 14: Enantioselektive Hydrosilylierung prochiraler Ketone mit $\mathrm{Rh}$ - und $\mathrm{Pt}$-Komplexen optisch

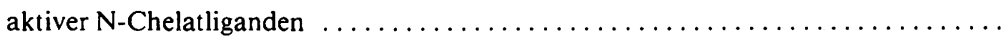

Hoffmann Ernst G., Nehl Hans, Lehmkuhl Herbert, Seevogel Klaus und Stempfle Werner: Untersuchungen zum strukturdynamischen Verhalten von 2-Alkenylzink-Verbindungen

Bogdanović Borislav, Liao Shih-tsien, Mynott Richard, Schlichte Klaus und Westeppe Uwe: Bildungsgeschwindigkeit und Charakterisierung von Magnesiumanthracen .........

Baudler Marianne, Saykowski Franz, Hintze Michael, Tebbe Karl-Friedrich, Heinlein Thomas, Vissers Antje und Fehér Magda: Beiträge zur Chemie des Phosphors, 131: Synthese und Eigenschaften der Methylendiphosphirane $(t-\mathrm{BuP}){ }_{2} \mathrm{C}=\mathrm{CR}_{2}(\mathrm{R}=\mathrm{H}, \mathrm{Me}$,

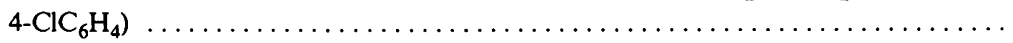

Keller Egbert und Wolters Doris: Benzonitril als 6-Elektronen-Donor-Ligand: Die Cluster $\mathrm{Fe}_{3}(\mathrm{CO})_{9}\left(\mu_{3}\left(\eta^{2}\right)-\mathrm{NCC}_{6} \mathrm{H}_{5}\right)$ und $\mathrm{Fe}_{4}(\mathrm{CO})_{12}\left(\mu_{4}\left(\eta^{2}\right)-\mathrm{NCC}_{6} \mathrm{H}_{5}\right) \ldots \ldots \ldots \ldots \ldots \ldots$

Roesky Herbert W., Lucas Joachim, Noltemeyer Mathias und Sheldrick George M.: (Disilylamino)phosphane $\left(\mathrm{R}_{\mathrm{f}}\right)_{2} \mathrm{P}-\mathrm{N}\left(\mathrm{SiMe}_{3}\right)_{2}-$ Bausteine für $\mathrm{PN}_{3} \mathrm{~S}_{2}$-Ringe $\ldots \ldots \ldots \ldots \ldots$

Brune Hans Albert, Unsin Josef, Alt Helmut G., Schmidtberg Günter und Spohn KarlHeinz: Kernspin-Kopplungskonstanten mit Platin-195 als Kriterien für die Konfigurations-Bestimmung platinorganischer Verbindungen des zweiwertigen Platins ........

\section{ORGANISCHE CHEMIE}

Heesing Albert und Laue Hans-Joachim: Wasserstoffübertragungen, 5: Zirkondioxid als

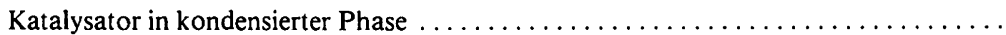

Schmidtchen Franz P.: Macrocyclische quartäre Ammoniumsalze, V: Katalyse aromatischer nucleophiler Substitutionen nach enzymanalogen Prinzipien $\ldots \ldots \ldots \ldots \ldots \ldots$

Kollenz Gert, Penn Gerhard, Dolenz Gerhard, Akcamur Yunus, Peters Karl, Peters EvaMaria und Schnering von Hans Georg: Untersuchungen von Reaktionsmechanismen durch Isotopenmarkierung, VIII: Zum Bildungsweg der Pyrrolo[2,3- $d$ ]pyrimidine aus 4-Benzoyl-5-phenyl-2,3-furandion und Arylisocyanaten . . . . . . . . . . . . .

Kollenz Gert, Penn Gerhard, Ott Walter, Peters Karl, Peters Eva-Maria und Schnering von Hans Georg: Reaktionen mit cyclischen Oxalylverbindungen, XXIII: Zur Reaktion heterocyclischer Fünfring-2,3-dione mit Carbodiimiden. - Eine Synthesemöglichkeit

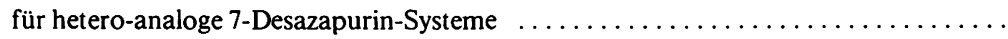

Brunner Henri, Reiter Barbara und Riepl Georg: Asymmetrische Katalysen, 14: Enantioselektive Hydrosilylierung prochiraler Ketone mit Rh- und Pt-Komplexen optisch aktiver $\mathrm{N}$-Chelatliganden 
Hollmann Günter und Vögtle Fritz: Kationen-Selektivität neuer Chromoionophore mit stark lösungsmittelabhängiger Lichtabsorption. Zur optischen Enantiomeren-Differenzierung mit chiralen Chromoionophoren $\ldots \ldots \ldots \ldots \ldots \ldots \ldots \ldots \ldots$

Hoffmann Ernst G., Nehl Hans, Lehmkuhl Herbert, Seevogel Klaus und Stempfle Werner: Untersuchungen zum strukturdynamischen Verhalten von 2-Alkenylzink-Verbin-

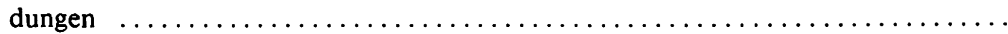

Bogdanović Borislav, Liao Shih-tsien, Mynott Richard, Schlichte Klaus und Westeppe Uwe: Schlichte Klaus und Westeppe Uwe: Bildungsgeschwindigkeit und Charak-

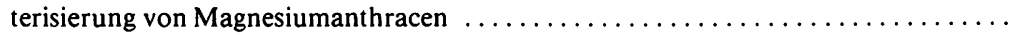

Carlsen Lars und Egsgaard Helge: Thermischer Zerfall von 1,2-Oxathiolan in der Gasphase

Schlegel Günter und Schäfer Hans J.: Anodische Oxidation von Organoborverbindungen

Flitsch Wilhelm und Lubisch Wilfried: Zur Umsetzung von 2-Pyrrolcarbaldehyd mit

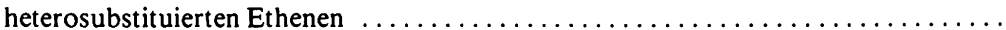

Langhals Elke, Langhals Heinz und Rüchardt Christoph: Der Nachweis eines Radikalketten-Mechanismus für die Knabe-Reaktion von 1,2-Dihydro-2-methylpapaverin . .

Berning Wilfried, Hünig Siegfried und Prokschy Frank: Azobrücken aus Azinen, III: Unerwartete Bildung einer Verbindung mit parallelen $\mathrm{C}=\mathrm{C}$ - und $\mathrm{N}=\mathrm{N}$-Bindungen .

Albert Bernhard, Berning Wilfried, Burschka Christian, Hünig Siegfried und Prokschy Frank: Azobrücken aus Azinen, IV: Intramolekulare [2+2]-Photocycloaddition zwischen parallelen $\mathrm{C}=\mathrm{C}$ - und $\mathrm{N}=\mathrm{N}$-Bindungen $\ldots \ldots \ldots \ldots \ldots \ldots \ldots \ldots \ldots \ldots \ldots$

Körösi Jenö, Láng Tibor, Sohár Pál, Neszmélyi András, Horváth Gyula und Zólyomi Gábor: Heterocyclische Verbindungen, VI: Bildung von Isomeren bei der Acylierung von Verbindungen mit 1-Aryl-4-methyl-5H-2,3-benzodiazepin-Skelett $\ldots \ldots \ldots \ldots$

Löhr Hans-Gerd, Josel Hans-Peter, Engel Aloys, Vögtle Fritz, Schuh Willi und Puff Heinrich: Einschlußverbindungen organischer Oniumsalze, IV: OrganylammoniumWirtsubstanzen als vielseitige Clathratbildner $\ldots \ldots \ldots \ldots \ldots \ldots \ldots \ldots \ldots$

Effenberger Franz und Beisswenger Thomas: Aminosäuren, 2: $N$-Acetyl- $\alpha, \beta$-didehydro$\alpha$-aminosäureester durch Stickstoffabspaltung aus $\alpha$-Azidocarbonsäureestern mit Rhenium-Katalysatoren

Beisswenger Thomas und Effenberger Franz: Aminosäuren, 3: $N$-Acetylierte $\alpha$, $\beta$-Didehydro- $\alpha$-aminosäure-Derivate durch Stickstoffabspaltung aus $\alpha$-Azidocarbonsäureamiden und $\alpha$-Azido- $\omega$-aminocarbonsäurelactamen mit Rhenium-Katalysatoren ...

Vorbrüggen Helmut und Krolikiewicz Konrad: Aminierung, III. - Trimethylsilanol als austretende Gruppe, V: Silylierung-Aminierung von Hydroxy-N-heterocyclen . .....

Binger Paul, McMeeking John und Schäfer Hannelore: Reaktionen der Cyclopropene, VI: Nickel(0)-katalysierte [2+1]-Cycloadditionen von 3,3-Diorganylcyclopropenen mit elektronenarmen Olefinen

Bestmann Hans Jürgen, Schmid Günter, Oechsner Helmut und Ermann Peter: Reaktionen mit Phosphinalkylenen, XLV: Reaktionen von Alkylidentriphenylphosphoranen mit Tetramethylformamidinium-chlorid. Synthese von [2-(Dimethylamino)vinyl]triphenylphosphonium-chloriden und (Formylalkyliden)triphenylphosphoranen .....

Quast Helmut und Saal von der Wolfgang: Mechanismus der säurekatalysierten Isomerisierung von trans- $N, N^{\prime}$-Dibenzyliden-1,2-cyclopropandiamin in cis-2,3-Dihydro-2,3- 
diphenyl-1 $H$-1,4-diazepin. cis-trans-Isomerisierung des intermediären $N$-Benzyliden1,2-cyclopropandiamins via Azomethin-ylid-Zwischenstufen $\ldots \ldots \ldots \ldots \ldots \ldots$

Allmann Rudolf, Kupfer Rainer, Nagel Michael und Würthwein Ernst-Ulrich: Zur Struktur von $N$-Methylencarbonsäureamiden: Röntgenstrukturergebnisse, spektroskopische Untersuchung und quantenmechanische Berechnungen $\ldots \ldots \ldots \ldots \ldots \ldots$

Brune Hans Albert, Unsin Josef, Alt Helmut G., Schmidtberg Günter und Spohn KarlHeinz: Kernspin-Kopplungskonstanten mit Platin-195 als Kriterien für die Konfigurations-Bestimmung platinorganischer Verbindungen des zweiwertigen Platins ...

Sucrow Wolfgang, Turnschek Wolfgang, Wolf Ulrich, d'Amour Hedwig und Krüger Carl: Enhydrazine, 36: Reaktion von Enhydrazinen mit Acetylencarbonsäureestern

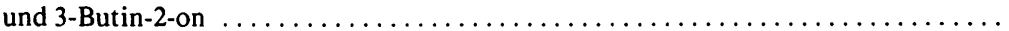

Goerdeler Joachim und Chuen-huei Ho: Dimere Isothiocyanate von Kohlensäureestern

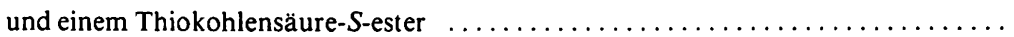

Neumeister Joachim und Griesbaum Karl: Anomale Produkte bei der erschöpfenden Ozonoxidation von Phenanthren in salzsaurem Methanol ..................

Kaupp Gerd: Thermische [2,1,3]-Eliminierung von Methanthiol aus einem 1,5-Benzothiazepin-Derivat ........................................... 1643

Berichtigung ............................................... 1647 


\section{INORGANIC CHEMISTRY}

Herrmann Wolfgang A., Bauer Christine, and Weichmann Josef: Transition Metal Methylene Complexes, XLIII: Three- and Four-membered Dimetallacycles Having Sulfur Dioxide, Selenium, Chlorogold, and a Silver Diazoalkane as Molecular Build-

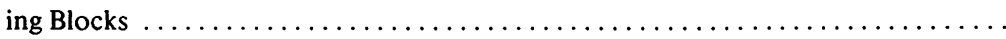

Brunner Henri, Reiter Barbara, and Riepl Georg: Asymmetric Catalyses, 14: Enantioselective Hydrosilylation of Prochiral Ketones with Rh- and Pt-Complexes of Optically

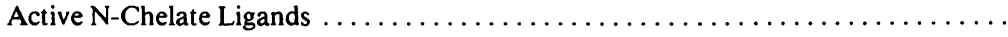

Hoffmann Ernst G., Nehl Hans, Lehmkuhl Herbert, Seevogel Klaus, and Stempfle Werner: Investigation of the Dynamic Structural Behavior of 2-Alkenylzinc Com-

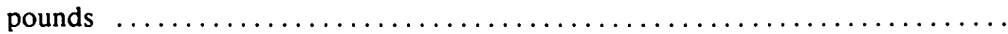

Bogdanović Borislaw, Liao Shih-tsien, Mynott Richard, Schlichte Klaus, and Westeppe Uwe: Rate of Formation and Characterization of Magnesium Anthracene ........

Baudler Marianne, Saykowski Franz, Hintze Michael, Tebbe Karl-Friedrich, Heinlein Thomas, Vissers Antje, and Fehér Magda: Contributions to the Chemistry of Phosphorus, 131: Synthesis and Properties of the Methylenediphosphiranes $(t-B u P)_{2} C=C_{2}$

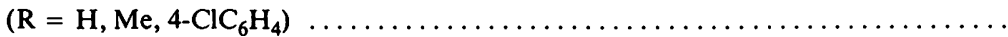

Keller Egbert and Wolters Doris: Benzonitrile as a 6-Electron Donor Ligand: The Cluster $\mathrm{Fe}_{3}(\mathrm{CO})_{9}\left(\mu_{3}\left(\eta^{2}\right)-\mathrm{NCC}_{6} \mathrm{H}_{5}\right)$ and $\mathrm{Fe}_{4}(\mathrm{CO})_{12}\left(\mu_{4}\left(\eta^{2}\right)-\mathrm{NCC}_{6} \mathrm{H}_{5}\right) \ldots \ldots \ldots \ldots \ldots \ldots$

Roesky Herbert W., Lucas Joachim, Noltemeyer Mathias, and Sheldrick George M.: (Disilylamino)phosphanes $\left(\mathrm{R}_{\mathrm{f}}\right)_{2} \mathrm{P}-\mathrm{N}\left(\mathrm{SiMe}_{3}\right)_{2}-$ Precursors for $\mathrm{PN}_{3} \mathrm{~S}_{2}$ Rings $\ldots \ldots \ldots$.

Brune Hans Albert, Unsin Josef, Alt Helmut G., Schmidtberg Günter, and Spohn KarlHeinz: Spin-Spin Coupling Constants by Platinum-195 as Criteria for a Configuration Determination in Platinum Organic Compounds

\section{ORGANIC CHEMISTRY}

Heesing Albert and Laue Hans-Joachim: Hydrogen Transfer Reactions, 5: The Catalytic Activity of Zirkonium Dioxide in Condensed Phase .....................

Schmidtchen Franz P.: Macrocyclic Quarternary Ammonium Salts, V: The Catalysis of Aromatic Nucleophilic Substitutions Following Enzyme-analogous Principles ......

Kollenz Gert, Penn Gerhard, Dolenz Gerhard, Akcamur Yunus, Peters Karl, Peters EvaMaria, and Schnering von Hans Georg: Mechanistic Investigations with the Aid of Isotopic Labeling, VIII: Investigations of the Reaction Pathway to Pyrrolo[2,3-d]pyrimidines from 4-Benzoyl-5-phenyl-2,3-furandione and Aryl Isocyanates . . . . . .

Kollenz Gert, Penn Gerhard, Ott Walter, Peters Karl, Peters Eva-Maria, and Schnering von Hans Georg: Reactions of Cyclic Oxalyl Compounds, XXIII: Reactions of Fivemembered 2,3-Dioxo Heterocycles with Carbodiimides - a Synthetic Pathway to

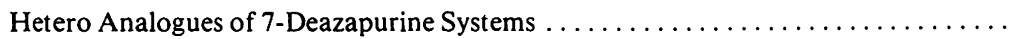

Brunner Henri, Reiter Barbara, and Riepl Georg: Asymmetric Catalyses, 14: Enantioselective Hydrosilylation of Prochiral Ketones with Rh- and Pt-Complexes of Optically Active N-Chelate Ligands 
Hollmann Günter and Vögtle Fritz: Cation Selectivity of New Chromoionophores Exhibiting Strong Solvent Depending Light Absorption. An Approach to Optical Enantiomer Differentiation Using Chiral Chromoionophores $\ldots \ldots \ldots \ldots \ldots \ldots \ldots \ldots$

Hoffmann Ernst G., Nehl Hans, Lehmkuhl Herbert, Seevogel Klaus, and Stempfle Werner: Investigation of the Dynamic Structural Behavior of 2-Alkenylzinc Compounds

Bogdanović Borislav, Liao Shih-tsien, Mynott Richard, Schlichte Klaus, and Westeppe Uwe: Rate of Formation and Characterization of Magnesium Anthracene ........

Carlsen Lars and Egsgaard Helge: Thermal Decomposition of 1,2-Oxathiolane in the Gas

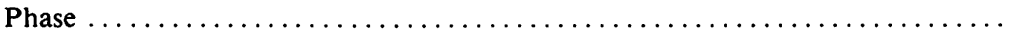

Schlegel Günter and Schäfer Hans J.: Anodic Oxidation of Organoboranes ...........

Flitsch Wilhelm and Lubisch Wilfried: On the Reaction of 2-Pyrrolecarbaldehyde with

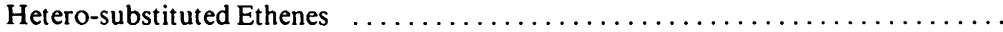

Langhals Elke, Langhals Heinz, and Rüchardt Christoph: Evidence for a Radical Chain Mechanism for the Knabe Reaction of 1,2-Dihydro-2-methylpapaverine ..........

Berning Wilfried, Hünig Siegfried, and Prokschy Frank: Azo Bridges from Azines, III: Unexpected Formation of a Compound with Parallel $C=C$ and $N=N$ Bonds .....

Albert Bernhard, Berning Wilfried, Burschka Christian, Hünig Siegfried, and Prokschy Frank: Azo Bridges from Azines, IV: Intramolekular [2 +2$]$ Photocycloaddition of

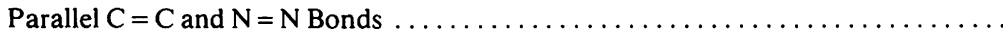

Körösi Jenö, Láng Tibor, Sohár Pál, Neszmélyi András, Horváth Gyula, and Zólyomi Gábor: Heterocyclic Compounds, VI: Formation of Isomers upon Acylation of Compounds Having a 1-Aryl-4-methyl-5H-2,3-benzodiazepine Skeleton . .........

Löhr Hans-Gerd, Josel Hans-Peter, Engel Aloys, Vögtle Fritz, Schuh Willi, and Puff Heinrich: Inclusion Compounds of Organic Onium Salts, IV: Organyl Ammonium Hosts as Versatile Clathrate Formers

Effenberger Franz and Beisswenger Thomas: Amino Acids, 2: $N$-Acetyl $\alpha, \beta$-Didehydro $\alpha$-Amino Acid Esters from $\alpha$-Azidocarboxylic Acid Esters and Acetic Anhydride by

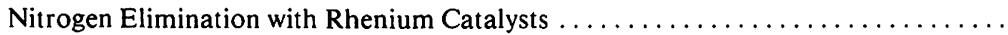

Beisswenger Thomas and Effenberger Franz: Amino Acids, 3: $N$-Acetylated $\alpha, \beta$-Didehydro $\alpha$-Amino Acid Derivatives by Nitrogen Elimination from $\alpha$-Azidocarboxylic Acid Amides and $\alpha$-Azido- $\omega$-aminocarboxylic Acid Lactams with Rhenium Catalysts

Vorbrüggen Helmut and Krolikiewicz Konrad: Amination, III. - Trimethylsilanol as Leaving Group, V: Silylation - Amination of Hydroxy N-Heterocycles . . . . . . .

Binger Paul, McMeeking John, and Schäfer Hannelore: Reactions of Cyclopropenes, VI: Nickel( 0$)$ Catalyzed [2+1]-Cycloadditions of 3,3-Diorganocyclopropenes with Electron-deficient Olefins . . . . . . . . . . . . . . . . . . . . . . . . . .

Bestmann Hans Jürgen, Schmid Günter, Oechsner Helmut, and Ermann Peter: Reactions with Phosphine Alkylenes, XLV: Reactions of Alkylidenetriphenylphosphoranes with Tetramethylformamidinium Chloride. Synthesis of [2-(Dimethylamino)vinyl]triphenylphosphonium Chloride and (Formylalkylidene)triphenylphosphoranes ....

Qua:t Helmut and Saal von der Wolfgang: Mechanism of the Acid-catalyzed Isomerization of trans- $N, N^{\prime}$-Dibenzylidene-1,2-cyclopropanediamine to cis-2,3-Dihydro-2,3- 
diphenyl-1 $H$-1,4-diazepine. cis-trans-Isomerization of Intermediate $\mathrm{N}$-Benzylidene-

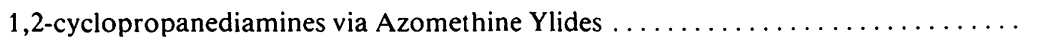

Allmann Rudolf, Kupfer Rainer, Nagel Michael, and Würthwein Ernst-Ulrich: On the Structure of $N$-Methylenecarboxamides: X-Ray Data, Spectroscopy, and Quantum

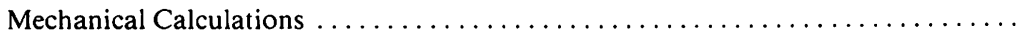

Brune Hans Albert, Unsin Josef, Alt Helmut G., Schmidtberg Günter, and Spohn KarlHeinz: Spin-Spin Coupling Constants by Platinum-195 as Criteria for a Configuration Determination in Platinum Organic Compounds

Sucrow Wolfgang, Turnschek Wolfgang, Wolf Ulrich, d'Amour Hedwig, and Krüger Carl: Enehydrazines, 36: Reaction of Enehydrazines with Acetylenecarboxylic Esters

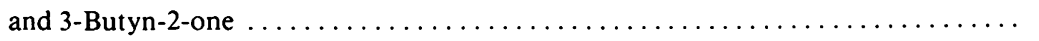

Goerdeler Joachim and Chuen-huei Ho: Dimeric Isothiocyanates of Carbonic Esters and

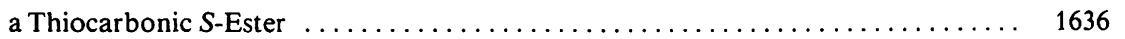

Neumeister Joachim and Griesbaum Karl: Abnormal Products from the Exhaustive Ozone Oxidation of Phenanthrene in $\mathrm{HCl}$ /Methanol . . . . . . . . . . . . . . . . . 1640

Kaupp Gerd: Thermal [2,1,3]-Elimination of Methanethiol from a 1,5-Benzothiazepine

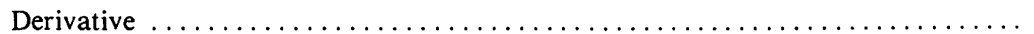

Correction 
Akcamur, Y. s. Kollenz, $G$.

Albert, B., Berning, W., Burschka, C.,

Hünig, S. und Prokschy, F.

1465

Allmann, R., Kupfer, R., Nagel, M. und

Würthwein, E.-U. 1597

Alt, H. G. s. Brune, H. A. 1606

Baudler, M., Saykowski, F., Hintze, M.,

Tebbe, K., Heinlein, T., Vissers, $A$.

und Fehér, $M$.

1542

Bauer, C. s. Herrmann, W. A. . . . . . . 1271

Beisswenger, $T$. und Effenberger, $F$. . 1513

- s. Effenberger, $F$. 1497

Berning, W., Hünig, S. und

Prokschy, F.

- s. Albert, $B$. 1465

Bestmann, H. J., Schmid, G.,

Oechsner, H. und Ermann, P. . . . 1561

Binger, P., McMeeking, J. und

Schäfer, $H$. 1551

Bogdanović, B., Liao, S., Mynott, R.,

Schlichte, K. und Westeppe, U. . . 1378

Brune, H. A., Unsin, J., Alt, H. G.,

Schmidtberg, G. und Spohn, K.-H. 1606

Brunner, H., Reiter, B. und Riepl, G. ... 1330

Burschka, C. s. Albert, B. ......... 1465

Carlsen, L. und Egsgaard, $H$.

Chuen-huei Ho s. Goerdeler, J. . . . . . . 1636

d'Amour, H. s. Sucrow, $W$.

1620

Dolenz, G. s. Kollenz, $G$.

Effenberger, $F$. und Beisswenger, $T$. . . 1497

- s. Beisswenger, $T$.

Egsgaard, $H$. s. Carlsen, $L$. 1393

Engel, A. s. Löhr, H.-G. 1487

Ermann, P. s. Bestmann, $H$. J.

Fehér, $M$. s. Baudler, $M$. 1542

Flitsch, $W$. und Lubisch, $W$. 1424

Goerdeler, J. und Chuen-huei Ho

1636

Griesbaum, K. s. Neumeister, $J$

1640

Heesing, $A$. und Laue, H.-J.

1263

Heinlein, T. s. Baudler, $M$.
Herrmann, W. A., Bauer, C. und

Weichmann, J. ........... 1271

Hintze, M. s. Baudler, $M$. . . . . . . 1542

Hoffmann, E. G., Nehl, H.,

Lehmkuhl, H., Seevogel, $K$. und

Stempfle, $W$. . . . . . . . . . 1364

Hollmann, G. und Vögtle, F. ....... 1355

Horváth, G. s. Körösi, J. . . . . . . . 1476

Hünig, S. s. Albert, B. . . . . . . . 1465

- s. Berning, W. . . . . . . . . . 1455

Josel, H.-P. s. Löhr, H.-G. . . . . . . . 1487

Kaupp, G. . . . . . . . . . . . . . 1643

Keller, E. und Wolters, D. . . . . . . 1572

Kettenring, J. K. s. Maas, G. . . . . . . 1647

Körösi, J., Lang, T., Sohár, P.,

Neszmélyi, A., Horváth, G. und

Zolyomi, $G$. 1476

Kollenz, G., Penn, G., Dolenz, G.,

Akcamur, Y., Peters, K., Peters, E.-M.

und Schnering, von, $H$. G.

-, Penn, G., Ott, W., Peters, K.,

Peters, E.-M. und

Schnering, von, $H . G$.

Krolikiewicz, K. s. Vorbrüggen, H. . . . 1523

Krüger, C. s. Sucrow, W. . . . . . . 1620

Kupfer, R. s. Allmann, R. . . . . . 1597

Láng, T. s. Körösi, J. . . . . . . . . 1476

Langhals, E., Langhals, $H$. und

Rüchardt, C. ........... 1436

Langhals, H. s. Langhals, E. . . . . . 1436

Laue, H.-J. s. Heesing, A. . . . . . . 1263

Lehmkuhl, H. s. Hoffmann, E. G. . . . 1364

Liao, S. s. Bogdanović, B. . . . . . . 1378

Löhr, H.-G., Josel, H.-P., Engel, A.,

Vögtle, F., Schuh, W. und Puff, H. 1487

Lubisch, W. s. Flitsch, $W$. . . . . . . 1424

Lucas, J. s. Roesky, H. W. . . . . . . 1583

Maas, G. und Kettenring, J. K. . . . . 1647

McMeeking, J. s. Binger, P. . . . . . 1551

Mynott, R. s. Bogdanović, B. ...... 1378 
Nagel, M. s. Allmann, R. . . . . . . 1597

Nehl, H. s. Hoffmann, E. G. . . . . . 1364

Neszmélyi, A. s. Körösi, J. .......... 1476

Neumeister, J. und Griesbaum, K. . . . . 1640

Noltemeyer, M. s. Roesky, H. W. .... 1583

Oechsner, H. s. Bestmann, H. J. . ... 1561

Ott, W. s. Kollenz, G.

1310

Penn, G. s. Kollenz, G. . . . . . . 1299, 1310

Peters, E.-M. s. Kollenz, G. . . . . 1299, 1310

Peters, K. s. Kollenz, G. . ...... 1299, 1310

Prokschy, F. s. Albert, B. ......... 1465

- s. Berning, $W$.

Puff, H. s. Löhr, H.-G. 1487

Quast, $H$. und Saal, von, der, $W$. 1591

Reiter, B. s. Brunner, $H$. 1330

Riepl, G. s. Brunner, $H$. 1330

Roesky, H. W., Lucas, J., Noltemeyer, M.

und Sheldrick, G. M. 1583

$R \ddot{u}$ chardt, C. s. Langhals, $E$. 1436

Saal, von, der, W. s. Quast, $H$. 1591

Saykowski, F. s. Baudler, $M$. 1542

Schäfer, $H$. s. Binger, $P$. 1551

Schäfer, H. J. s. Schlegel, G. 1400

Schlegel, G. und Schäfer, H. J. ...... 1400

Schlichte, $K$. s. Bogdanovic, $B$. 1378

Schmid, G. s. Bestmann, H. J.
Schmidtberg, G. s. Brune, H. A. .... 1606

Schmidtchen, F. P. .............. 1287

Schnering, von, $H$. G.

s. Kollenz, $G$. 1299,1310

Schuh, W. s. Löhr, H.-G. ......... 1487

Seevogel, K. s. Hoffmann, E. G. ..... 1364

Sheldrick, G. M. s. Roesky, H. W. . . . 1583

Sohár, P. s. Körösi, J. ........... 1476

Spohn, K.-H. s. Brune, H. A. . . ... 1606

Stempfle, W. s. Hoffmann, E. G. . ... 1364

Sucrow, W., Turnschek, W., Wolf, U., d'Amour, $H$. und Krüger, $C$. $\ldots 1620$

Tebbe, K. s. Baudler, M. . .......... 1542

Turnschek, W. s. Sucrow, W. . . . . . 1620

Unsin, J. s. Brune, H. A. . . . . . . . 1606

Vissers, A. s. Baudler, M. . . . . . . . 1542

Vögtle, F. s. Hollmann, G. ....... 1355

- s. Löhr, H.-G. ................. 1487

Vorbrüggen, $H$. und Krolikiewicz, K. .. 1523

Weichmann, J. s. Herrmann, W. A. . . 1271

Westeppe, U. s. Bogdanović, B. ...... 1378

Wolf, U. s. Sucrow, W. . . . . . . . . 1620

Wolters, D. s. Keller, E. . . . . . . . . 1572

Würthwein, E.-U. s. Allmann, R. . ... 1597

Zólyomi, G. s. Körösi, J. . . .......... 1476 


\title{
Der Nachweis eines Radikalketten-Mechanismus für die Knabe-Reaktion von 1,2-Dihydro-2-methylpapaverin ${ }^{1)}$
}

\author{
Elke Langhals, Heinz Langhals und Christoph Rüchardt* \\ Institut für Organische Chemie und Biochemie der Universität Freiburg, \\ Albertstr. 21, D-7800 Freiburg i. Br.
}

Eingegangen am 29. Juni 1983

Die Knabe-Reaktion von 1,2-Dihydro-2-methylpapaverin (9) und die begleitende Eliminierungsreaktion wurden durch ihre gebrochene Reaktionsordnung und durch die Möglichkeit der Inhibition als Radikalkettenreaktionen erkannt, deren kettentragendes Radikal das 3,4-Dimethoxybenzylradikal ist. Die Synthese von $N$-Methylpavin (19) aus 1,2-Dihydro-2-methylpapaverin (9) ist an die Anwesenheit von Ameisensäure als Inhibitor der Radikalketten gebunden. Durch Zusatz von Inhibitoren können nun Immoniumionen 10 und analoge Verbindungen stabilisiert und ihre Chemie untersucht werden.

\section{Evidence for a Radical Chain Mechanism for the Knabe Reaction of} 1,2-Dihydro-2-methylpapaverine ${ }^{1)}$

A free radical chain mechanism is proposed for the Knabe reaction and the accompanying elimination reaction on account of their nonintegral reaction order and of successful inhibition experiments. 3,4-Dimethoxybenzyl radicals are the chain carrying species. Battersby's synthesis of $N$-methylpavine (19) from 1,2-dihydro-2-methylpapaverine (9) is dependent on the presence of formic acid as radical chain inhibitor. In the presence of inhibitors 1-benzyl-1,4-dihydro-2-methylisoquinolinium ions like $\mathbf{1 0}$ are persistent species whose chemistry can now be investigated.

Die 1963 entdeckte Knabe-Reaktion 2), auch 1,2-Dihydroisochinolin-Umlagerung genannt, stellt eine Methode zur Darstellung der nicht als Naturstoffe auftretenden 3-Benzylisochinoline aus den in der Natur bekannten 1-Benzylisochinolinen dar. Bei Einwirkung von Säure werden 2-Alkyl-1-benzyl-1,2-dihydroisochinoline 1 vermutlich über ein intermediär gebildetes Immoniumion 2 in 2-Alkyl-3-benzyl-3,4-dihydroisochinoliniumionen 3 umgelagert ${ }^{3)}$. Wegen der Oxidationsempfindlichkeit von 1 und 2 wird die Knabe-Reaktion im allgemeinen unter $\mathrm{N}_{2}$ und ohne Isolierung der Zwischenstufe $\mathbf{2}$ in situ durchgeführt.

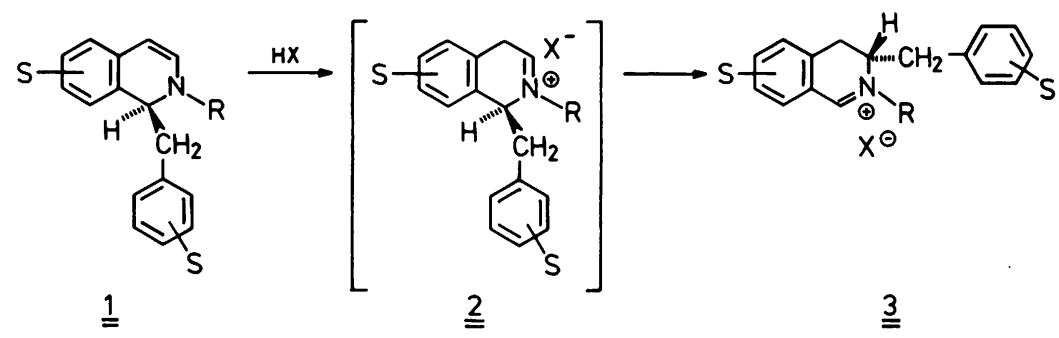

(C) Verlag Chemie GmbH, D-6940 Weinheim, 1984

0009 - 2940/84/0404-1436\$02.50/0 
Triebkraft der Reaktion dürfte die Ausbildung eines konjugierten Systems in $\mathbf{3}$ sein, das man im Vergleich zu 2 auch an der bathochromen Verschiebung der längstwelligen Absorptionsbande im UV-Spektrum erkennt. Trotz eingehender Bearbeitung 3,4) ist der Mechanismus dieser Reaktion bis heute ungeklärt. Während die der Benzylwanderung $2 \rightarrow 3$ entsprechende Wanderung von Allyl- und substituierten Allylresten als intramolekular ablaufende Cope-analoge [3,3]-sigmatrope Umlagerung erkannt wurde ${ }^{5)}$, wiesen Kreuzungsexperimente für die Benzylwanderung $\mathbf{2} \rightarrow \mathbf{3}$ einen intermolekularen Verlauf nach ${ }^{6}$. Kinsman und Dyke kamen durch kinetische Messungen zu dem Schluß, daß es sich um eine Reaktion 2. Ordnung handelt. Wegen der stark negativen Aktivierungsentropie von $-43.1 \mathrm{cal} / \mathrm{mol} \cdot \mathrm{K}$ postulierten sie einen bimolekular verlaufenden synchronen Austauschmechanismus ${ }^{4}$, der allerdings die Regeln der Orbitalsymmetrie verletzte ${ }^{7)}$. Stereochemische Experimente widerlegten ihn schließlich. Knabe und Mitarbb. stellten nämlich fest, daß die Benzylwanderung bevorzugt antarafacial verläuft (siehe Formulierung $1 \rightarrow 3$ ), was

Schema 1

\section{Start}<smiles>[R]N1C=Cc2ccccc2C1Cc1ccccc1</smiles>

$\stackrel{1}{=}$<smiles>CCCCCC</smiles><smiles>[R]N1C=CC2=CC=CCC2C1</smiles>

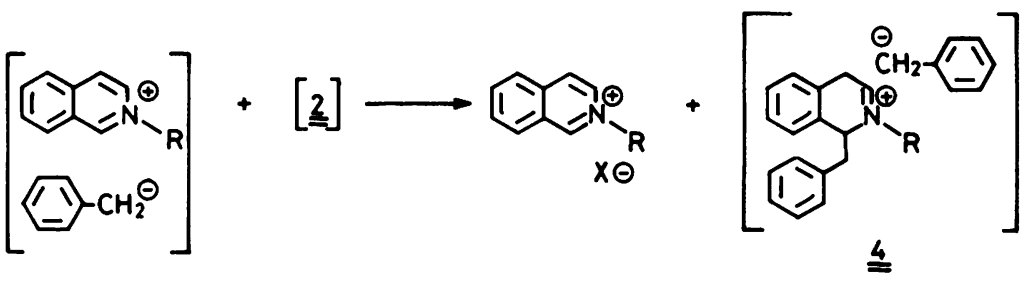

Kette<smiles>[R]N1CCc2ccccc2C1c1ccccc1CCc1ccccc1</smiles>

$\underline{4}$

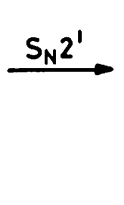

$+[\underline{\underline{2}}] \longrightarrow \underline{3}+[\underline{4}]$<smiles>[R][N+]1([2H])Cc2ccccc2CC1Cc1ccccc1</smiles>

$\underline{\underline{5}}$

Abbruch

$$
\underline{\underline{4}} / \underline{\underline{5}}+\mathrm{HX} \longrightarrow \underline{\underline{2}} / \underline{\underline{3}}+\longrightarrow \mathrm{CH}_{3}
$$

Chem. Ber. $117(1984)$ 
mit dem bimolekularen Austauschmechanismus ${ }^{4}$ ) nicht vereinbar ist ${ }^{8}$. Nach Diskussion verschiedener weiterer intermolekular verlaufender Varianten kamen Knabe und Heckmann ${ }^{3)}$ zum Vorschlag eines über Kontaktionenpaare verlaufenden anionischen Kettenmechanismus (siehe Schema 1), der, zumindest qualitativ, den meisten experimentellen Befunden gerecht wurde.

Als Start der Kette sollte durch Ionisation des nicht protonierten Enamins 1 ein Kontaktionenpaar entstehen, das mit dem Immonium-Ionenpaar 2 das Anion austauscht unter Bildung des Ionenpaars 4. Dieses geht im ersten Kettenschritt die eigentliche $S_{N} 2^{\prime}$-Reaktion zu 5 unter formalem Benzylanionen-Austausch ein. Durch erneuten Anionen-Austausch zwischen den Ionenpaaren 5 und 2 entsteht im zweiten Kettenschritt das Produkt 3 und ein erneut in die Kette eingehendes Ionenpaar 4. Als Kettenabbruch wird die Bildung von Toluol durch Hydrolyse von 4 oder 5 postuliert ${ }^{3)}$. Die von diesem Mechanismus geforderte Bildung von 2-Alkylisochinoliniumionen (siehe 2. Startschritt) und von Toluol (siehe Kettenabbruch) ist als Nebenreaktion bzw. Konkurrenzreaktion der Knabe-Reaktion seit langem bekannt ${ }^{3)}$.

Die Crux dieses mechanistischen Vorschlags ist 1. das Postulat nicht dissoziierender Kontaktionenpaare im wäßrig-alkoholischen, also polaren Medium, 2. das Postulat des Anionenaustauschs zwischen Kontaktionenpaaren, während Winstein hierfür solvensgetrennte Ionenpaare forderte 9), und 3. das Auftreten von Benzylcarbanionen im wäßrig-sauren Medium ohne deren spontane und vollständige Protonierung.

In Kenntnis der in neuerer Zeit bekannt gewordenen variationsreichen Radikalchemie unter Beteiligung von Aminium-Radikalkationen ${ }^{10}$ ) und der hohen Reaktivität nucleophiler Alkyl- und Benzylradikale bei Additionsreaktionen an elektronenarme $\pi$-Systeme $\left.{ }^{10 b}, 11\right)$, hielten wir einen radikalischen Kettenmechanismus für wahrscheinlicher, bei dem im 1. Schritt durch Addition eines Benzylradikals an das Immoniumsalz 2 ein Aminium-Radikalkation 6 gebildet wird, das im 2.

\section{Radikalkette}

1)

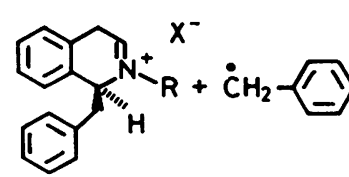

$\underline{\underline{2}}$

2)
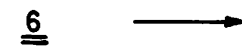

\section{Abbruch}
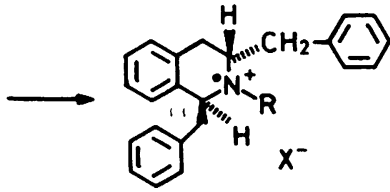

$\underline{6}$

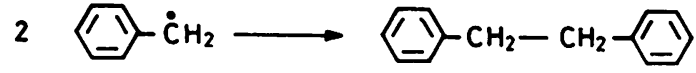

Kettenschritt in bekannter Reaktion ${ }^{10)}$ von der 1-Position ein Benzylradikal abspaltet unter Bildung des Produktes 3. Eine Analogie zum 1. Schritt dieser Kette konnten wir inzwischen in der photochemischen Addition von Iodbenzol ${ }^{12)}$ an $N, N$-Dimethyl-methylenimmoniumionen auffinden.

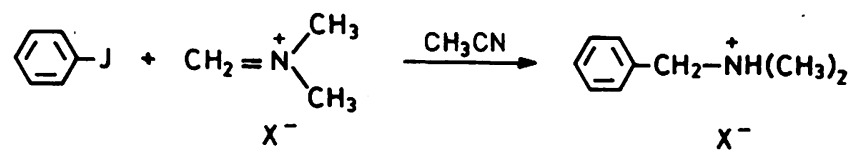

Chem. Ber. 117(1984) 
Diesem Vorschlag, der früher schon ähnlich diskutiert ${ }^{3,4)}$ aber verworfen wurde, stand entgegen, daß die früheren Bearbeiter weder durch ESR noch durch CIDNP den Radikalnachweis erfolgreich führen konnten ${ }^{3,4)}$. Da die postulierten Kettenschritte nach unserer Schätzung sehr schnell verlaufen und zu großer Kettenlänge führen sollten, halten wir für diesen Fall die beiden genannten spektroskopischen Methoden zur Prüfung auf radikalische Zwischenstufen für nicht geeignet. Für das Auftreten einer Radikalkette gab es bereits einzelne Hinweise. Bibenzyl wurde als Nebenprodukt, wenn auch in sehr geringer Menge $(\leqq 0.2 \%)^{4)}$ nachgewiesen, womit sich der Kettenabbruch zwanglos formulieren läßt. Es wurden gelegentlich Induktionsperioden beobachtet, für die der anwesende Luftsauerstoff verantwortlich gemacht wurde ${ }^{4,13)}$. Einige analog über Radikalketten verlaufende 1,3-Umlagerungen sind bekannt ${ }^{4,14,15)}$. Schließlich verläuft die als Radikalkette formulierte Thioallylumlagerung ${ }^{15)}$ ebenfalls mit antarafacialer Stereochemie wie sie für $\mathbf{1} \rightarrow \mathbf{3}$ gefunden wurde. Nach unseren Erfahrungen ist auch die stark negative Aktivierungsentropie Anlaß, einen Radikalketten-Mechanismus zu vermuten ${ }^{16)}$.

Um dieses Postulat zu prüfen, führten wir eine mechanistische Studie der Titelreaktion durch, wobei wir die Möglichkeit der Inhibition der Radikalkette als wichtigstes Kriterium ansahen.

\section{Synthesen und Produktbilanz}

Zur Synthese der Modellverbindung 1,2-Dihydro-2-methylpapaverin (9) methylierten wir Papaverin (7) im Gegensatz zur Lit. ${ }^{13,17)}$ ohne Solvens mit einem Überschuß an Methyliodid, wobei direkt und quantitativ das reine kristallisierte $N$-Methylderivat 8 unter Rückgewinnung des überschüssigen Methyliodids gewonnen wurde. 9 erhält man aus 8 durch Reduktion mit $\mathrm{LiAlH}_{4}$. Während die früheren Bearbeiter 9 und analoge Verbindungen wegen deren Oxidationsempfindlichkeit direkt als etherische Lösung aus dem Reduktionsschritt in die Knabe-Reaktion einsetzten, reinigten wir 9 durch Umlösen aus Ether. Reines kristallisiertes 9 erwies sich vor Licht geschützt als beständig und bei $25^{\circ} \mathrm{C}$ lagerfähig. Es wurden korrekte Werte der Elementaranalyse erhalten, und 9 wurde für alle im folgenden beschriebenen Versuche kristallisiert eingesetzt.

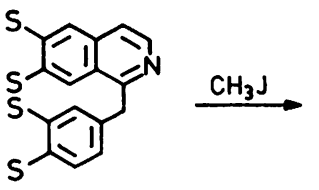

$\stackrel{7}{=}$

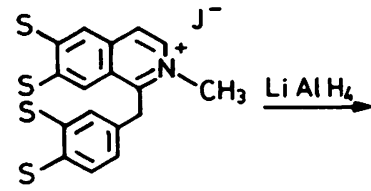

$\underline{\underline{8}}$

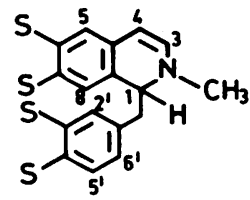

$\underline{\underline{9}}$

$$
\mathrm{S}=\mathrm{CH}_{3} \mathrm{O}-
$$

Abb. 1 zeigt das hochaufgelöste ${ }^{1}$ H-NMR-Spektrum von 9 , in dem sämtliche aromatische und vinylische Protonen aufgelöst sind. Das Signal von 8-H ist deutlich durch die diamagnetische Wirkung der Dimethoxybenzylgruppe in 1-Position zu hohem Feld verschoben ${ }^{18)}$ und klar von den anderen aromatischen Protonensignalen abgesetzt. Die erkennbare long-range-Kopplung zwischen $\mathrm{H}-1$ und $\mathrm{H}-3^{18,19)}$ fehlt im $3-\mathrm{H}$-Signal des Spektrums des mit LiAlD ${ }_{4}$ erhaltenen [1-D]-1,2-Dihydro-2-methylpapaverins ([1-D]-9). 

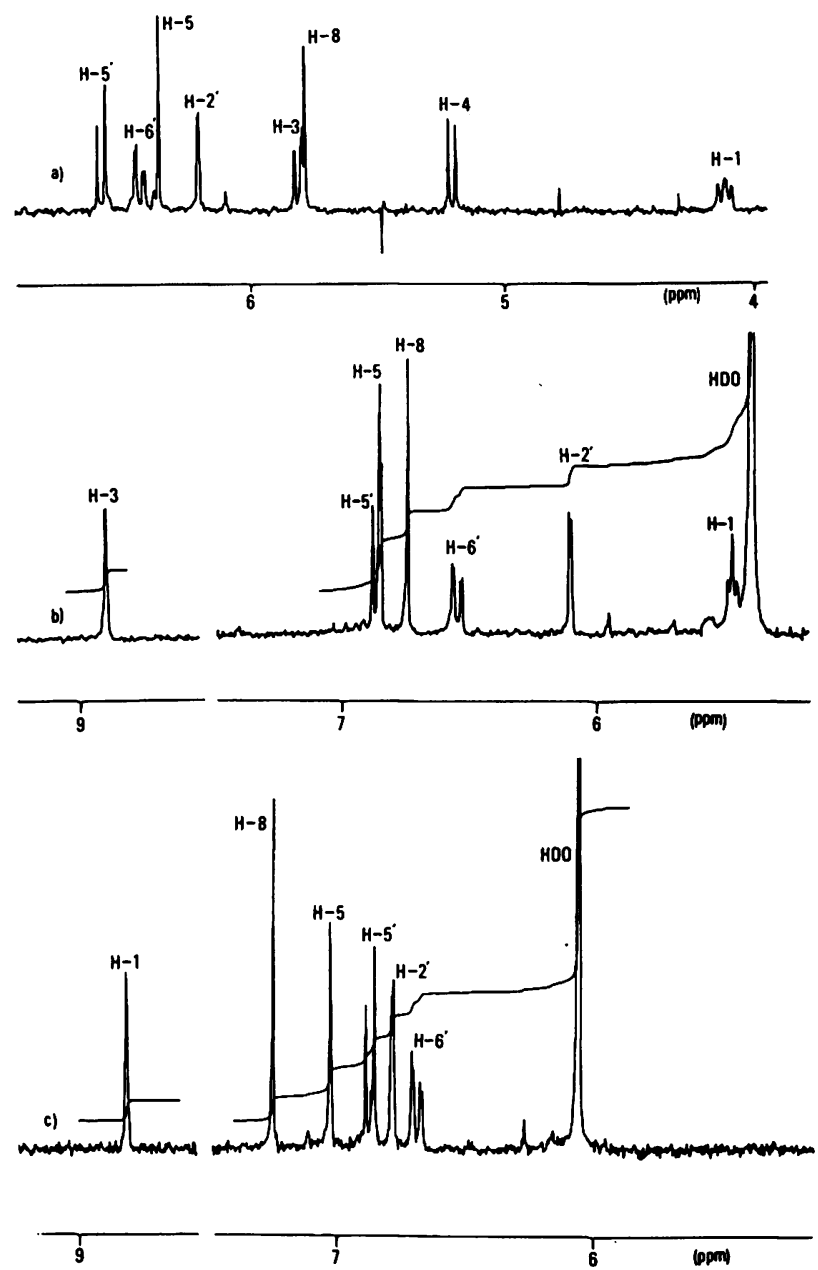

Abb. 1. Ausschnitte aus den hochaufgelösten ${ }^{1} \mathrm{H}-\mathrm{NMR}$-Spektren $(250 \mathrm{MHz})$ von a) 9 in $\left[D_{6}\right]$ Benzol/ $\left[D_{12}\right]$ Cyclohexan, b) 10 in $2 \mathrm{~N} \mathrm{DCl} / \mathrm{CD}_{3} \mathrm{OD}$ unter Zusatz von Tribromessigsäure (4-Position bisdeuteriert) und c) 11 in $2 \mathrm{~N} \mathrm{DCl} / \mathrm{CD}_{3} \mathrm{OD}$

Die in salzsaurer Lösung ablaufende Umlagerung $1 \rightarrow 3$ bzw. $9 \rightarrow 11$ wird üblicherweise bei $60-100^{\circ} \mathrm{C}$ mit Reaktionszeiten von $1 \mathrm{~h}$ und darüber vorgenommen ${ }^{8)}$ und durch Abtrennen des Produktes 3 bzw. 11a mit NaCN als „Pseudocyanid“, z. B. 14, aufgearbeitet. Aus letzterem erhält man mit Perchlorsäure das Perchlorat von 3 bzw. 11b. Durch Messung der UV-Absorption während der Reaktion stellten wir fest, daß 9 bei strengem Ausschluß von Sauerstoff durch Arbeiten unter $\mathrm{N}_{2}$ in $4.07 \cdot 10^{-2} \mathrm{M}$ Lösung in einem Gemisch von Ethanol und $2 \mathrm{~N} \mathrm{HCl}$ bei $20^{\circ} \mathrm{C}$ schon nach $1 \mathrm{~h}$ vollständig zu $11 \mathrm{a}$ isomerisiert war. Nach Einengen der Lösung im Vakuum ließ sich mit Perchlorsäure direkt das reine Perchlorat 11 b ausfällen. 


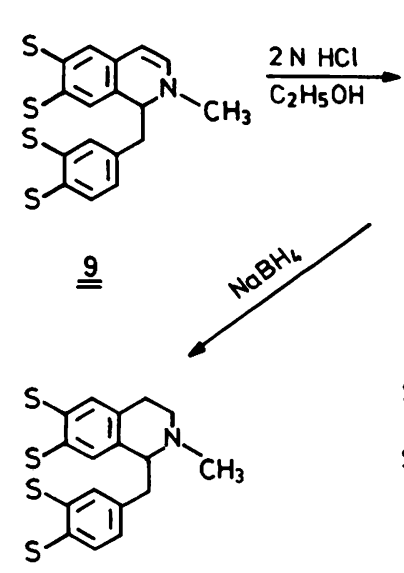

$\underline{\underline{12}}$<smiles></smiles>

$\underline{\underline{10}}$

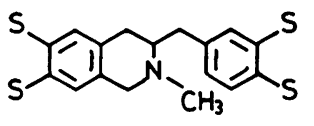

$\stackrel{13}{=}$

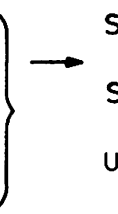<smiles>[X][N+]1(C)Cc2cc(S)c(S)cc2C[C@H]1Cc1ccc(S)c(S)c1</smiles>
II a) $x=\mathrm{Cl}^{-}$ b) $x^{-}=\mathrm{ClO}^{-}$ $U V\left(\mathrm{CH}_{3} \mathrm{OH}\right)=\lambda_{\max }(\lg \varepsilon)=368 \mathrm{~nm}(3.94)$

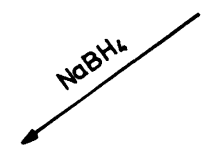<smiles>CC1(C)c2cc(S)c(S)cc2CC1Cc1ccc(S)c(S)c1</smiles>

$\stackrel{14}{=}$

\section{$\mathrm{S}=\mathrm{CH}_{3} \mathrm{O}-$}

Zur Prüfung, ob die Isomerisierung $9 \rightarrow 11$ a quantitativ verläuft, bedienten wir uns des Verfahrens der Aufarbeitung, das Kinsman und $D y k e^{4)}$ für ihre kinetischen Messungen ausgearbeitet hatten. 9 wurde bei 21 bzw. $46^{\circ} \mathrm{C}$ in Ethanol/Salzsäure (1:1) unter $\mathrm{N}_{2}$ umgesetzt. Die in Abständen entnommenen Proben wurden mit $\mathrm{NaHCO}_{3}$ neutralisiert und mit $\mathrm{NaBH}_{4}$ reduziert. Nach Ansäuern und Einengen i. Vak. erhielt man nach Extraktion mit $\mathrm{CH}_{2} \mathrm{Cl}_{2}$ Gemische von 12 und 13. Vergleichsproben dieser beiden Verbindungen waren auf dem gleichen Weg aus reinem 9 und 11 dargestellt worden. Die Analyse des Aromatenbereichs der hochaufgelösten ${ }^{1} \mathrm{H}$-NMR-Spektren ergab, daß bei der Knabe-Umlagerung von 9 und der anschließenden $\mathrm{NaBH}_{4}$-Reduktion maximal 5\% an Nebenprodukten entstanden waren ${ }^{1)}$. Die Spektren von 12 und 13 unterscheiden sich charakteristisch und eignen sich daher für die quantitative Produktanalyse. Auffallend ist vor allem die Hochfeldverschiebung des 8-H zugeordneten Signals von $12 \mathrm{im}$ Vergleich zu dem von 13. Wie oben bereits für 9 diskutiert, ist dies auf den diamagnetischen Ringstromeffekt der 1-ständigen 3,4-Dimethoxybenzylgruppe zurückzuführen ${ }^{18,20)}$. Da in den Spektren der Produktgemische 12 und 13 alle Signale diesen beiden Komponenten zugeordnet werden konnten, läuft die Knabe-Reaktion offensichtlich fast quantitativ zu 11 ab. Durch die Isolierung und erkannte Lagerfähigkeit der Ausgangsverbindung 9 und den Nachweis des quantitativen Verlaufs der KnabeReaktion $9 \rightarrow \mathbf{1 1}$ waren die Voraussetzungen für eine kinetische Studie geschaffen.

Obwohl sich die geschilderte Arbeitsweise von Kinsman und Dyke $e^{4)}$ - sie verfolgten das Konzentrationsverhältnis 12:13 durch GC - für kinetische Messungen eignet, arbeiteten wir eine Methode aus, bei der die Reaktion direkt ohne Aufarbeitung spektroskopisch verfolgt werden kann, in der Hoffnung, Präzision und Schnelligkeit der Messungen zu erhöhen.

\section{Kinetische Messungen}

Die Knabe-Reaktion von 9 ließ sich durch Messen der Zunahme der Extinktion von 11 bei $368 \mathrm{~nm}$ quantitativ verfolgen ${ }^{8 a)}$. Wegen des hohen Extinktionskoeffizienten am 
Absorptionsmaximum ( $\lg \varepsilon=3.94)$ mußten Proben gezogen und vor der Messung verdünnt werden, wofür sich Methylcellosolve als geeignet erwies ${ }^{1)}$. Da wir festgestellt hatten, daß die Isomerisierung unterhalb einer Konzentration von $c_{0} \approx 2 \cdot 10^{-3} \mathrm{~mol} \cdot \mathrm{l}^{-1}$ praktisch unmeßbar langsam wird, war die Reaktion in den Proben durch die Verdünnung bereits gestoppt. Effizienter und präziser als dieses Verfahren war jedoch die direkte und kontinuierliche UV-spektroskopische Verfolgung der Reaktion im langwellig abklingenden Ast der längstwelligen Absorptionsbande von 11. Mit Hilfe der Tangentenmethode ${ }^{21)}$ ließ sich aus der Bandenform im Bereich von $\lambda_{\max }$ berechnen, daß für die beabsichtigten Messungen bei $436 \mathrm{~nm}$ der optimale Extinktionskoeffizient auftritt. Die Gültigkeit des Lambert-Beerschen Gesetzes wurde geprüft und bestätigt. In Tab. 1 sind nur die kontinuierlich bei $436 \mathrm{~nm}$ durchgeführten kinetischen Messungen aufgeführt. Sie stimmen mit den allerdings etwas stärker streuenden diskontinuierlich durch Probenverdünnung erhaltenen im Rahmen der Meßgenauigkeit überein "). Die Auswertung wurde nach der 1., 1.5. und 2. Ordnung vorgenommen. Nach den Korrelationskoeffizienten in Tab. 1 zu urteilen, ist bei den höheren Anfangskonzentrationen das Zeitgesetz der 1.5. Ordnung am besten erfüllt, während bei niedrigerer Konzentration die 2. Ordnung den besten Korrelationskoeffizienten gibt.

Tab. 1. Kinetik der Knabe-Reaktion von 1,2-Dihydro-2-methylpapaverin (9) in Ethanol/2 $\mathrm{N} \mathrm{HCl}$ $(1: 1)$ bei $20^{\circ} \mathrm{C}$ (spektroskopisch bei $436 \mathrm{~nm}$ gemessen)

\begin{tabular}{|c|c|c|c|c|c|}
\hline $\mathrm{Nr}$. & $c_{0} \cdot 100^{\text {a) }}$ & $\begin{array}{l}\text { Anzahl der } \\
\text { Meßpunkte }\end{array}$ & $\begin{array}{l}\text { Reaktions- } \\
\text { ordnung }\end{array}$ & $\left.k \cdot 10^{4} \mathrm{c}\right)$ & $r^{\mathrm{d})}$ \\
\hline 1 & 4.07 & 24 & $\begin{array}{l}1 \\
1.5 \\
2\end{array}$ & $\begin{array}{c}7.90 \\
61.2 \\
488\end{array}$ & $\begin{array}{l}0.9943 \\
0.9993 \\
0.9965\end{array}$ \\
\hline 2 & 2.57 & 23 & $\begin{array}{l}1 \\
1.5 \\
2\end{array}$ & $\begin{array}{c}7.01 \\
62.6 \\
570\end{array}$ & $\begin{array}{l}0.9946 \\
0.9996 \\
0.9987\end{array}$ \\
\hline 3 & 2.03 & 24 & $\begin{array}{l}1 \\
1.5 \\
2\end{array}$ & $\begin{array}{c}7.32 \\
80.0 \\
898\end{array}$ & $\begin{array}{l}0.9870 \\
0.9979 \\
0.9993\end{array}$ \\
\hline 4 & 1.25 & 23 & $\begin{array}{l}1 \\
1.5 \\
2\end{array}$ & $\begin{array}{l}4.05 \\
56.6 \\
812\end{array}$ & $\begin{array}{l}0.9880 \\
0.9980 \\
0.9996\end{array}$ \\
\hline
\end{tabular}

a) Startkonzentration an $9\left(\mathrm{~mol} \cdot \mathrm{1}^{-1}\right)$, berechnet aus der Einwaage. - b) Vorgegebene Reaktionsordnung bei der Auswertung. - c) Errechnete Geschwindigkeitskonstanten nach der jeweiligen Reaktionsordnung in $\mathrm{s}^{-1}, 1^{0.5} \cdot \mathrm{mol}^{-0.5} \cdot \mathrm{s}^{-1}$ bzw. $1 \cdot \mathrm{mol}^{-1} \cdot \mathrm{s}^{-1}$, Fehler ca. 5\%. - d) Korrelationskoeffizient.

Zur weiteren Prüfung dieser Aussage wurde die Reaktionsordnung in der Zeit nach Van't Hoff ${ }^{22)}$ bestimmt, indem man die Extinktions-Zeit-Kurven der bei $c_{0}=2.57$. $10^{-2} \mathrm{~mol} \cdot 1^{-1}$ ausgeführten Messung mit Hilfe der Normalen graphisch differenzierte und $\ln v=\ln \mathrm{d} E / \mathrm{d} t$ gegen den Umsatz $\ln \left(E_{\infty}-E\right)$ auftrug ${ }^{22)}$. Es zeigte sich dann in Abb. 2, daß die der Reaktionsordnung entsprechende Steigung der Kurve nicht konstant ist, sondern von anfangs $\approx 1.5$ mit zunehmendem Umsatz auf $\approx 2$ ansteigt. Hierdurch wird verständlich, daß Dyke et al. ${ }^{4)}$ die 2 . Ordnung bestimmten. Aus ihren Anga- 
ben ist zu entnehmen, daß die Auswertung der Messungen erst nach ca. 50\% Umsatz begonnen worden war. Die von uns festgestellte gebrochene Reaktionsordnung und deren Ansteigen mit dem Umsatz ist ein gutes Indiz für den Ablauf eines Kettenmechanismus ${ }^{23)}$.

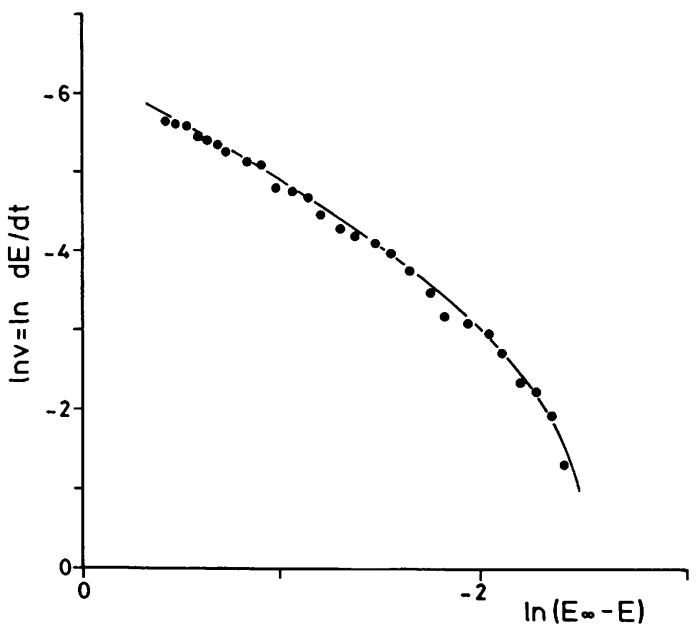

Abb. 2. Bestimmung der Reaktionsordnung der Reaktion $\mathbf{9} \rightarrow \mathbf{1 1}$ in der Zeit nach Van't Hoff ${ }^{22}$ ) für $c_{0}=2.57 \cdot 10^{-2} \mathrm{~mol} \cdot 1^{-1}$ (siehe Tab. 1). $E_{\infty}=0.762$

\section{Inhibitionsversuche}

Der beste Beleg für eine Radikalkette ist ihre Inhibition. Die Schwierigkeit, für die Knabe-Umlagerung einen geeigneten Inhibitor zu finden, liegt in der Kombination der an diesen zu stellenden Anforderungen. So muß er in Ethanol/2 $\mathrm{N} \mathrm{HCl} \mathrm{löslich} \mathrm{und}$ beständig sein, und er darf weder mit dem Edukt noch mit dem Produkt der Reaktion reagieren. Er darf also weder oxidierende noch reduzierende Eigenschaften besitzen. Deshalb ist z. B. die früher erwähnte Ausbeutereduktion an 11 bei Zusatz des oxidierend wirkenden Porphyrexids ${ }^{24,13)}$ zu 9 in Eisessig/Methanol nicht eindeutig zu interpretieren. Knabe et al. ${ }^{8,13)}$ hatten auch bereits festgestellt, daß Luftsauerstoff und besonders Einleiten von Sauerstoff die Umlagerungsfunktion unterdrückt. Wegen der bereits erwähnten Oxidationsempfindlichkeit des Edukts der Reaktion ist auch dieser Befund nur durch ein quantitatives Studium deutbar. Wir haben deshalb den Einfluß von Sauerstoff auf die Reaktionsgeschwindigkeit und den Umsatz der Reaktion von 9 untersucht (siehe Tab. 2). Unter Bedingungen, die unter $\mathrm{N}_{2}$ zu $91 \% 11$ führten, waren unter Sauerstoff lediglich maximal $12 \%$ entstanden. Durch GC-MS-Analyse waren unter Verwendung von Vergleichsproben geringe Mengen an 3,4-Dimethoxytoluol (16), 3,4-Dimethoxybenzyl-ethyl-ether $(17)^{25)}$ und 3,4-Dimethoxybenzaldehyd (18) nachweisbar, die vermutlich aus 3,4-Dimethoxybenzylradikalen (15) durch $\mathrm{H}$-Übertragung bzw. Abreaktion zum Hydroperoxid mit bekannten Folgereaktionen entstehen.

Chem. Ber. 117 (1984) 
Tab. 2. Inhibition der Knabe-Reaktion von 9 durch verschiedene Radikalfänger in Ethanol/2 $\mathrm{N} \mathrm{HCl}(1: 1)$ unter $\mathrm{N}_{2}$ (weitere Parameter sind den jeweiligen Erfordernissen angepaßt)

\begin{tabular}{|c|c|c|c|c|c|c|c|}
\hline $\begin{array}{l}\text { Versuch } \\
\text { Nr. }\end{array}$ & $\begin{array}{l}\text { Temp. } \\
\left({ }^{\circ} \mathrm{C}\right)\end{array}$ & $\begin{array}{c}\left.c_{0} \cdot 100^{\mathrm{a}}\right) \\
\left(\mathrm{mol} \cdot 1^{-1}\right)\end{array}$ & Inhibitor & $\begin{array}{c}\left.c_{1} \cdot 100^{\mathrm{b}}\right) \\
\left(\mathrm{mol} \cdot 1^{-1}\right)\end{array}$ & $\begin{array}{l}\text { Best.- } \\
\text { Methode }^{c}\end{array}$ & $\begin{array}{c}\left.\text { Umsatzd }^{d}\right) \\
(\%)\end{array}$ & $\begin{array}{l}\text { Umsatze) } \\
\text { (ber. } \% \text { ) }\end{array}$ \\
\hline 1 & $21.0^{f)}$ & 2.69 & Sauerstoff & g) & NMR & $<12(70)^{\mathrm{h})}$ & 91 \\
\hline 2 & $21.0^{f)}$ & 1.38 & $\mathrm{Br}_{3} \mathrm{C}-\mathrm{CO}_{2} \mathrm{H}$ & 4.23 & $\left.N \mathbf{R}^{i}\right)$ & $0(180)$ & $70^{\mathrm{j})}$ \\
\hline 3 & $20.0^{f)}$ & 2.10 & 3-Cyanpyridin & 18.85 & UVk) & $0(210)$ & 98 \\
\hline 4 & $19.5^{f)}$ & 1.41 & 3-Cyanpyridin & 9.91 & NMR & $0(70)$ & 86 \\
\hline 5 & $19.5^{f)}$ & 1.32 & 3-Cyanpyridin & 0.58 & NMR & $26(70)$ & 85 \\
\hline 6 & $18.5^{f)}$ & 1.87 & Diethylether & 643 & UVk) & $0(450)$ & 99 \\
\hline 7 & $20.0^{f)}$ & 1.40 & Diethylether & 397 & NMR & $0(70)$ & 85 \\
\hline 8 & $80^{1)}$ & 1.51 & Diethylether & m) & NMR & $70(70)$ & - \\
\hline 9 & $85^{1)}$ & 2.81 & Diethylether & m) & NMR & $90(300)$ & - \\
\hline 10 & $20.0^{f)}$ & 2.45 & Ameisensäure & n) & $\left.N{ }^{i}{ }^{i)}, U^{k}\right)$ & $0(1080)$ & 100 \\
\hline 11 & $85^{1)}$ & 43.6 & Ameisensäure & o) & NMR & $0(240)^{p)}$ & - \\
\hline
\end{tabular}

a) Startkonzentration von 9. - b) Konzentration des Inhibitors. - c) Beschreibung der Methoden s. Text. - d) Reaktionszeiten (min) in Klammern; $0 \%$ bedeutet, daß kein Umlagerungsprodukt nachweisbar ist, Nachweisgrenze ca. 3\%. - e) Berechneter Umsatz einer nicht inhibierten Reaktion, hierbei wurde eine Ordnung von 1.5 zugrunde gelegt mit einem aus den Werten von Tab. 1 gemittelten $\vec{k}=65.1 \cdot 10^{-4} \mathrm{l}^{0.5} \cdot \mathrm{mol}^{-0.5} \cdot \mathrm{s}^{-1}$. $\left.-\mathrm{f}\right) \pm 0.2 \mathrm{grd}$. g) Unter Zutritt von Luftsauerstoff. - h) Oberer Grenzwert, da die Absorptionsbande bei $\lambda_{\max }=368 \mathrm{~nm}$ für 11 als Schulter auf einer kürzerwelligen Nebenproduktbande liegt. - i) Gaschromatographisch Spuren von 1,2,3,4-Tetrahydro-6,7-dimethoxy-2-methylisochinolin nachweisbar. - j) Im Vergleichsexperiment ohne Inhibitor bestimmter Umsatz. - k) UV-spektroskopisch Spuren von 6,7-Dimethoxy-2-methylisochinolinium-chlorid nachweisbar. 1) \pm 2 grd. - m) $2 \mathrm{~N} \mathrm{HCl} /$ Ethanol (1:1), ethergesättigt bei $20^{\circ} \mathrm{C}$. - ${ }^{n)}$ In Ameisensäure $/ 2 \mathrm{~N} \mathrm{HCl} /$ Ethanol $(2: 1: 1)$. - o) In Ameisensäure/2 N HCl (7:1). p) Bildung von $N$-Methylpavin $(19,64.5 \%)$ 


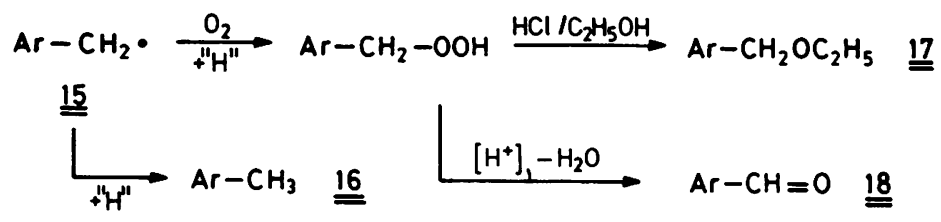

$A r=3,4-$ Dimethoxyphenyl

Knabe und Powilleit ${ }^{13)}$ hatten aus der Umlagerung des 1,2-Dihydro-6,7-dimethoxy2-methyl-1-(1-phenylethyl)isochinolins in $2 \mathrm{~N} \mathrm{HCl}$ entsprechend Ethylbenzol, 1-Phenylethanol und Acetophenon nachgewiesen.

Übersichtlicher verliefen Inhibitionsversuche mit Tribromessigsäure ${ }^{26)}$ (siehe Tab. 2). Zusatz von 3 Äquivalenten dieser Polyhalogenverbindung ${ }^{27)}$ inhibierte die KnabeReaktion von 9 über $3 \mathrm{~h}$ vollständig, wie durch NMR-Analyse und durch Aufarbeitung mit $\mathrm{NaBH}_{4}$ gezeigt wurde. Ohne Inhibitor waren unter sonst gleichen Reaktionsbedingungen im Parallelversuch 70\% 11 erhalten worden. Durch Zusatz von Tribromessigsäure ließ sich auch erstmals in einer Lösung von 9 in $2 \mathrm{~N} \mathrm{DCl} / \mathrm{CD}_{3} \mathrm{OD}(1: 1)$ das ${ }^{1} \mathrm{H}-$ NMR-Spektrum des Immoniumions 10 aufnehmen (siehe Abb. 1b), das selbst nach $3 \mathrm{~h}$ noch ungestört registriert werden konnte. Ohne Inhibitor ist bei der für NMRMessungen nötigen Konzentration die Knabe-Reaktion viel zu schnell für eine spektroskopische Untersuchung von $\mathbf{1 0}$.

Die kaum aufgelöste Aufspaltung des Signals für 3-H kommt durch long-rangeKopplung mit der $N$-Methylgruppe zustande, die selbst als Dublett erscheint, und nicht durch Kopplung mit 1-H (siehe Abb. 1 b). Dies wurde durch das unveränderte Auftreten dieser Aufspaltung im Spektrum der in 1-Position deuterierten Verbindung belegt. Aus der Tatsache, daß keine Signale der Methylenprotonen in 4-Position im Spektrum auftreten, ist zu folgern, daß an dieser Stelle vollständiger H/D-Austausch eingetreten ist. Das Gleichgewicht $\mathbf{9} \rightleftarrows \mathbf{1 0}$ stellt sich also schnell und vollständig ein.

Als guter Inhibitor der Knabe-Reaktion von 9 erwies sich auch 3-Cyanpyridin, das in saurer Lösung protoniert vorliegt und dann besonders schnell mit Alkyl- und Benzylradikalen reagiert ${ }^{28)}$. Setzt man der Standardreaktionslösung von Tab. 2 3-Cyanpyridin im Überschuß zu, so ist selbst nach 3.5 h UV-spektroskopisch noch kein Umsatz zu 11 feststellbar. Selbst 0.44 Moläquivalent 3-Cyanpyridin wirken noch deutlich inhibierend. Die Feststellung, daß man erst mit äquivalenten Mengen der genannten Inhibitoren vollständige Unterdrückung der Reaktion erreicht, spricht dafür, daß beide Kettenschritte äußerst schnell ablaufende Reaktionen sind. In weiteren Versuchen wurde auch eine hemmende Wirkung von Diethylether festgestellt (Tab. 2). Hierfür dürfte dessen bekannt hohe Reaktivität mit Aminiumradikalkationen verantwortlich sein ${ }^{10 a, b)}$. Bei Erhitzen auf $80-85^{\circ} \mathrm{C}$ wird diese Inhibierung wirkungslos, wofür vermutlich das Abdampfen des Ethers und dessen Wirkung als Schutzgas verantwortlich ist. Dies erklärt auch, warum frühere Autoren ${ }^{2)}$ das durch $\mathrm{LiAlH}_{4}$-Reduktion in Ether gewonnene 9 nach Zusatz von Salzsäure durch Erhitzen und Abdampfen des Ethers auf dem Wasserbad erfolgreich zur Umsetzung brachten.

Im Gegensatz zu diesen Inhibitoren erwiesen sich $\mathrm{BrCCl}_{3}$ und Hydrochinon in äquivalenten Mengen zugesetzt als unwirksam ${ }^{1)}$. Die Ursache dafür wurde nicht weiter un- 
tersucht, sie könnte in wirksamer Kettenübertragung liegen. Auf die inhibierende Wirkung der Ameisensäure wird im nächsten Abschnitt eingegangen (siehe Tab. 2).

\section{Pavinbildung und Eliminierung als Nebenreaktionen}

Als Nebenreaktion der Knabe-Umlagerung von 9 wurde bereits die zu 2-Methylisochinoliniumionen und Toluol führende Eliminierung erwähnt, deren Mechanismus unter dem Eindruck des radikalischen Verlaufs der Hauptreaktion auch neu überdacht werden muß. Einen Anstoß zur Klärung dieser Frage erhielten wir durch das Studium der von Battersby und Binks beschriebenen Synthese des $N$-Methylpavins ${ }^{29)}$ (19). Durch 25 stdg. Erhitzen von 9 auf $120^{\circ} \mathrm{C}$ in einem Gemisch von Orthophosphorsäure und 80proz. Ameisensäure entsteht 19 mit 76\% Ausbeute, ohne Störung durch die Knabe-Reaktion.

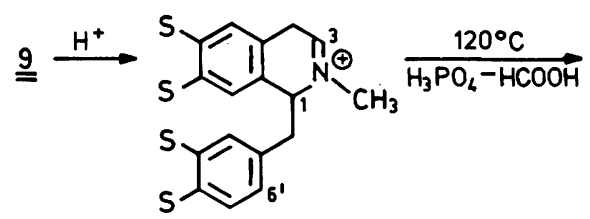

$\stackrel{10}{=}$

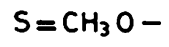

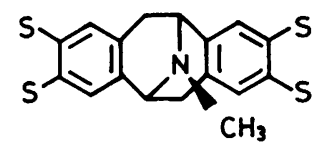

$\underline{\underline{19}}$

Hierbei handelt es sich offenbar um eine elektrophile Substitution in 6'-Position durch das elektrophile C-3 der Immoniumgruppierung in 10. Diese Synthese mutet um so erstaunlicher an, als Dyke und Mitarbb. ${ }^{30)}$ durch Knabe-Umlagerung von 9 in $2 \mathrm{~N}$ $\mathrm{H}_{3} \mathrm{PO}_{4}$ bei $100^{\circ} \mathrm{C}$ in hoher Ausbeute 11 erhalten hatten. Erst bei starker Herabsetzung der Anfangskonzentration an 9 und Erhöhen der Temperatur auf $155^{\circ} \mathrm{C}$ trat als Nebenreaktion auch $N$-Methylpavinbildung ein. Diese widersprüchlich scheinenden Befunde lassen sich nun einfach erklären.

Die Pavinbildung ist eine intramolekulare, also nach der 1. Ordnung verlaufende elektrophile Substitution mit, wie üblich, großem Temperaturkoeffizienten der Reaktionsgeschwindigkeit. Die Geschwindigkeit der konkurrierenden Knabe-Reaktion wird wegen ihres Radikalketten-Charakters weniger von der Temperatur aber um so stärker von der Konzentration erhöht (1.5-2. Ordnung). Kleine Aktivierungsenthalpien $\Delta H^{\ddagger}$ und negative Aktivierungsentropien $\Delta S^{*}$ sind typisch für Kettenreaktionen ${ }^{31)}$. Bei den Versuchen von Battersby war offenbar die Ameisensäure von entscheidender Bedeutung. Da Formylgruppen gute H-Donatoren sind ${ }^{32)}$ - vor allem gegenüber elektronegativen Radikalen ${ }^{32 d}$ - , vermuten wir, daß Ameisensäure als Inhibitor der KnabeReaktion wirkt und somit der Pavinbildung den Weg freigibt. Die beiden letzten Versuche in Tab. 2 bestätigen dies. Bei $20^{\circ} \mathrm{C}$ wird die Knabe-Reaktion durch Zusatz von Ameisensäure völlig inhibiert, und 10 konnte selbst nach $18 \mathrm{~h}$ noch durch NMR oder $\mathrm{NaBH}_{4}$-Reduktion und übliche Aufarbeitung als unverbraucht nachgewiesen werden. Bereits bei $85^{\circ} \mathrm{C}$ erhält man aber $65 \% \mathrm{~N}$-Methylpavin (19) als farblose kristallisierte Reinsubstanz durch übliche Aufarbeitung.

Um zu prüfen, ob die Ameisensäure die Radikalkette durch $\mathrm{H}$-Übertragung auf das 3,4-Dimethoxybenzylradikal 15 inhibiert, setzten wir $\mathrm{DCO}_{2} \mathrm{H}$ ein und prüften durch 
GC-MS-Analyse den Isotopengehalt des in geringer Menge entstandenen 3,4-Dimethoxytoluols (16). Dieses war frei von Deuterium.

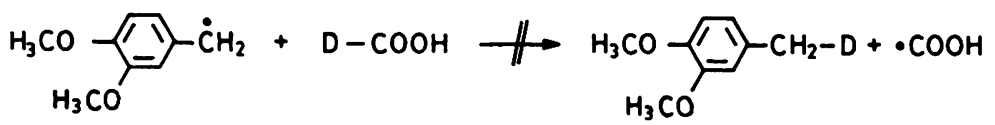

$\underline{\underline{15}}$

Vermutlich fängt die Ameisensäure also das 6 entsprechende Aminium-Radikalkation durch $\mathrm{H}$-Übertragung $\mathrm{ab}$, weil dieses als stark elektronegative Spezies bei der $\mathrm{H}$-Übertragung von Ameisensäure besonders reaktiv sein sollte.

Führte man die Knabe-Reaktion von 9 in einem Kontrollversuch in $\mathrm{D}_{2} \mathrm{O} / \mathrm{HCO}_{2} \mathrm{D}$ durch, so fand man durch GC-MS-Analyse entgegen der Erwartung monodeuteriertes 3,4-Dimethoxytoluol $([\alpha-\mathrm{D}]-16)^{33)}$. Da weder die Bildung von 3,4-Dimethoxybenzylanionen wahrscheinlich war, noch das Solvens als qualifizierter $\mathrm{H}$-Überträger in Frage kommt, nehmen wir an, daß [4- $\left.D_{2}\right]-10$ der D-Überträger ist. Es ist ja oben gezeigt worden, daß 10 unter den Bedingungen der Knabe-Reaktion in deuteriertem Solvens in 4-Position vollständigen H/D-Austausch erfährt. Diese D-Übertragung aus der 4-Position von $\mathbf{1 0}$ halten wir für die Schlüsselreaktion zur Erklärung der begleitenden Eliminierung, die wir ebenfalls als Radikalkette formulieren.

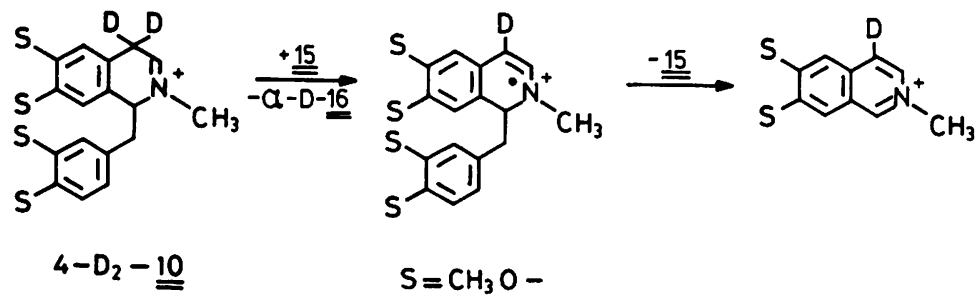

Die zur Eliminierung führende $\mathrm{H}$-Übertragung aus der 4-Position von 10 konkurriert vor allem dann erfolgreich mit der Addition des Benzylradikals an das Bindungssystem des Immoniumions in 10, die zur Knabe-Umlagerung führt, wenn das Benzylradikal elektronegative Substituenten trägt ${ }^{34)}$ oder protoniert ist, wie bei den $\alpha$-Picolylradikalen $^{35)}$. 1,2-Dihydro-2-methyl-1-picolylisochinolin geht im Sauren ausschließlich die Eliminierungsreaktion ein. Es ist verständlich, daß die H-Übertragung aus der 4-Position von 10 auf substituierte Benzylradikale den kleineren negativen $\rho$-Wert hat als die Addition in 3-Stellung von 10. Der zweite Schritt der Eliminierungskette ist nichts anderes als die Umkehrreaktion des 1. Schrittes einer Minisci-Alkylierung des Isochinoliniumions ${ }^{28,10 b}$ ). In Abwesenheit von Redoxkatalysatoren ist dieser Schritt sicher umkehrbar ${ }^{10 b)}$.

\section{Diskussion und Zusammenfassung}

Auf der Basis der beschriebenen experimentellen Befunde läßt sich der mechanistische Verlauf der Knabe-Reaktion und ihrer Neben- bzw. Konkurrenzreaktionen in einer Übersicht zusammenfassen.

Chem. Ber. 1/7(1984) 


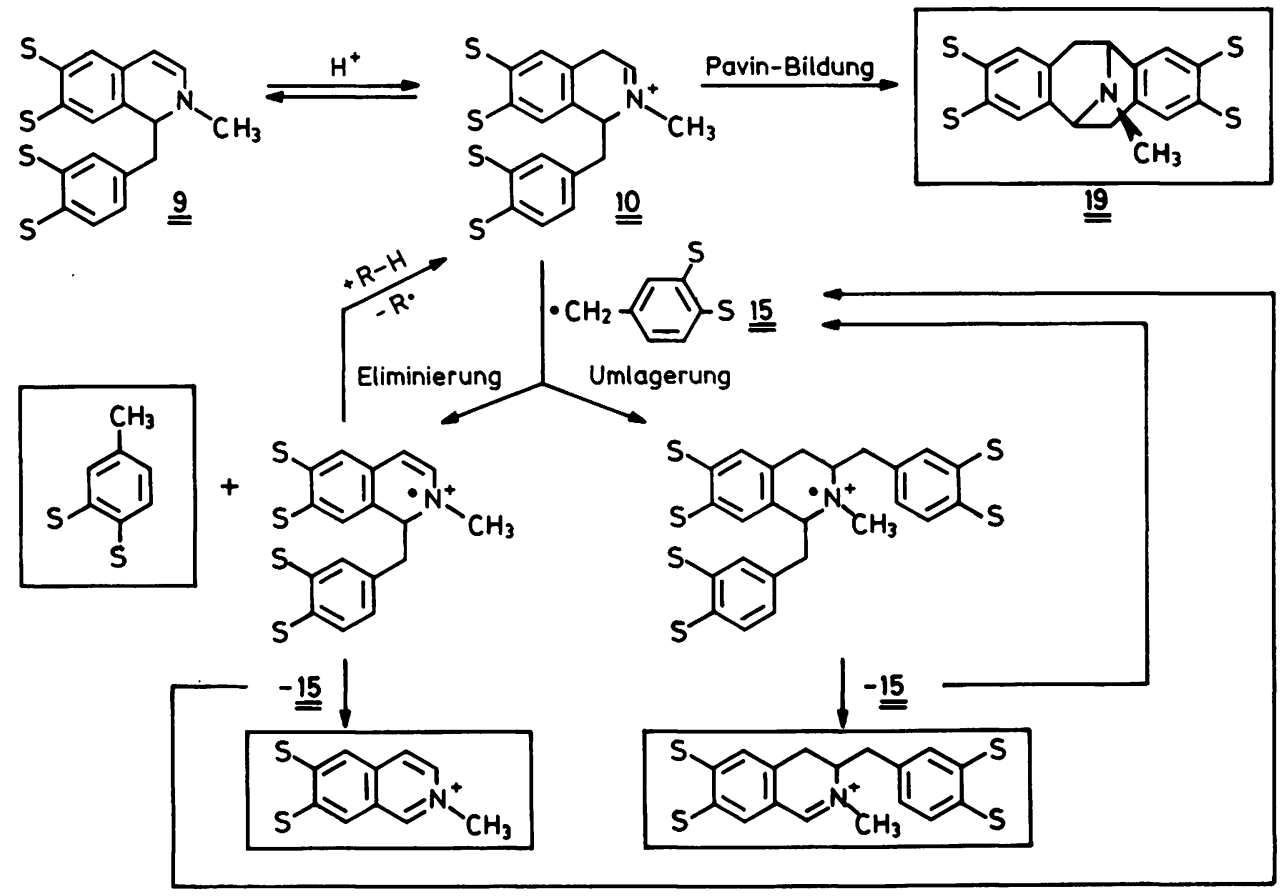

Kettenabbruch :

2<smiles>[CH2]c1ccc(S)c(S)c1</smiles>

$\underline{\underline{15}}$

Über den Start der Radikalkette ist zwar wenig bekannt. Es ist denkbar, daß ein Autoxidationsprozeß durch Spuren von Sauerstoff eine Rolle spielt und dabei z. B. Immoniumion 10 in 4-Stellung zum Aminium-Radikalkation oxidiert wird. Das 3,4-Dimethoxybenzylradikal 15 ist sowohl bei der Kette der Knabe-Umlagerung als auch bei der Eliminierungskette das kettentragende Radikal. Die Konkurrenz dieser beiden Reaktionen hängt daher von der Elektronegativität des beteiligten Benzylradikals ab. Als Kettenabbruch kommt die Bildung von 3,3',4,4'-Tetramethoxybibenzyl in Frage, das bereits früher nachgewiesen worden ist ${ }^{4}$. $N$-Methylpavin (19) entsteht aus 10 bei erhöhter Temperatur, wenn die Kette durch Inhibitoren oder durch Verdünnung nicht meßbar schnell ablaufen kann. Das Vorliegen einer effizienten Radikalkette erklärt auch das Ausbleiben des CIDNP-Effekts und das Fehlen von ESR-Signalen, denn Radikale treten dabei nur in geringster Konzentration auf und Radikalpaare allenfalls beim Kettenabbruch.

Die Kenntnis des Mechanismus sollte es nun erleichtern, Knabe-Reaktionen zu optimieren: sie sollten bei Raumtemperatur in Abwesenheit von Inhibitoren wie Sauerstoff und Ether und mit hohen Eduktkonzentrationen durchgeführt werden. Die Inhibierung 
der Knabe-Reaktion und die damit erreichte Stabilisierung von Immoniumionen des Typs 10, die bislang immer spontan isomerisierten, sollte es nun ermöglichen, die Chemie dieser Spezies z. B. bei Mannich-Reaktionen zu untersuchen.

Herrn Prof. Dr. J. Knabe gilt unser Dank für informative Diskussionen. Dem Fonds der Chemischen Industrie danken wir für die Förderung, Herrn Dr. D. Hunkler für die Aufnahme der NMR-Spektren und Herrn Dr. J. Wörth für die der Massenspektren.

\section{Experimenteller Teil}

Verwendete Geräte: IR-Spektrometer 457 der Fa. Perkin-Elmer; UV/VIS-Spektrometer: DMR 21 und PMQ III der Fa. Zeiss; NMR-Spektrometer: EM 390 der Fa. Varian und WM 250 der Fa. Bruker (TMS als interner Standard); GC-MS-Kopplungen: Massenspektrometer MAT 44S der Fa. Finnigan-MAT; Schmelzpunkte: Apparatur nach Dr. Tottoli der Fa. Büchi.

2-Methylpapaverinium-iodid [1-(3,4-Dimethoxybenzyl)-6,7-dimethoxy-2-methylisochinoliniumiodid, 8]: $18.1 \mathrm{~g}$ (53.2 mmol) Papaverin [1-(3,4-Dimethoxybenzyl)-6,7-dimethoxyisochinolin, 7] erwärmt man mit $120 \mathrm{ml}$ Methyliodid unter Licht-, Feuchtigkeits- und Sauerstoffausschluß ca. $3 \mathrm{~h}$ auf $50^{\circ} \mathrm{C}$. Die erkaltete Mischung wird abgesaugt. Die blaßgelben Kristalle wäscht man mit Ether und trocknet sie i. Vak. über Phosphorpentoxid. Überschüssiges Methyliodid gewinnt man aus der Mutterlauge destillativ zurück. Ausb. quantitativ (NMR-spektroskopisch Methyliodid-Spuren nachweisbar). Schmp. $135-138^{\circ} \mathrm{C}$ (Zers.), Lit. ${ }^{36)} 135-136^{\circ} \mathrm{C}$. - IR (KBr): $2930 \mathrm{w}(\mathrm{C}-\mathrm{H})$; $1605 \mathrm{~m}(\mathrm{C}-\mathrm{C}) ; 1565 \mathrm{~m} \mathrm{~cm}^{-1}$. - ${ }^{1} \mathrm{H}-\mathrm{NMR}\left(\mathrm{CDCl}_{3}\right): \delta=3.63\left(\mathrm{~s}, \mathrm{OCH}_{3}\right), 3.69\left(\mathrm{~s}, \mathrm{OCH}_{3}\right), 3.87$ $\left(\mathrm{s}, \mathrm{OCH}_{3}\right), 4.00\left(\mathrm{~s}, \mathrm{OCH}_{3}\right), 4.38\left(\mathrm{~s}, \mathrm{NCH}_{3}\right), 4.97\left(\mathrm{~s}, \mathrm{CH}_{2}\right), 6.13\left(\mathrm{dd}, \mathrm{J}_{65}=8 \mathrm{~Hz}, J_{62}=2.4 \mathrm{~Hz}\right.$, $\left.6^{\prime}-\mathrm{H}\right), 6.53\left(\mathrm{~d}, J_{65}=8 \mathrm{~Hz}, 5^{\prime}-\mathrm{H}\right), 6.87\left(\mathrm{~d}, J_{62}=2.4 \mathrm{~Hz}, 2^{\prime}-\mathrm{H}\right), 7.38(\mathrm{~s}, 8-\mathrm{H}), 7.57(\mathrm{~s}, 5-\mathrm{H}), 8.17(\mathrm{~d}$, $\left.J_{43}=6.6 \mathrm{~Hz}, 3-\mathrm{H}\right), 8.57\left(\mathrm{~d}, J_{43}=6.6 \mathrm{~Hz}, 4-\mathrm{H}\right)$.

1,2-Dihydro-2-methylpapaverin [1-(3,4-Dimethoxybenzyl)-1,2-dihydro-6,7-dimethoxy-2-methylisochinolin, 9]: $4.81 \mathrm{~g} \mathrm{(10} \mathrm{mmol)} 8$ werden in $200 \mathrm{ml}$ absol. Ether mit $0.76 \mathrm{~g}$ (20 mmol) Lithiumaluminiumhydrid unter $\mathrm{N}_{2}$-Schutzgas reduziert. Die Aufarbeitung gemäß Lit. ${ }^{4}$ ) liefert beim Ein-

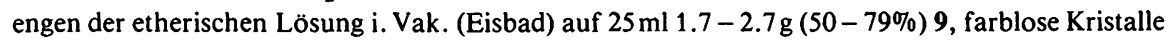
aus Ether, Schmp. $121^{\circ} \mathrm{C}$ (Zers.), Lit. ${ }^{4)} 122^{\circ} \mathrm{C}$. - IR (KBr): $2940 \mathrm{~m}, 2835 \mathrm{w}(\mathrm{C}-\mathrm{H}) ; 1620 \mathrm{~m}$ (Schulter, $\mathrm{C}=\mathrm{N}$ oder $\mathrm{C}=\mathrm{C}$ ) $\mathrm{cm}^{-1}$. - ${ }^{1} \mathrm{H}-\mathrm{NMR}$ ( $\left[\mathrm{D}_{6}\right]$ Benzol/ $\left[\mathrm{D}_{12}\right]$ Cyclohexan): $\delta=2.65-2.85$ $\left(\mathrm{m}, \mathrm{CH}_{2}\right), 2.73\left(\mathrm{~s}, \mathrm{NCH}_{3}\right), 3.40\left(\mathrm{~s}, \mathrm{OCH}_{3}\right), 3.49\left(\mathrm{~s}, \mathrm{OCH}_{3}\right), 3.61\left(\mathrm{~s}, \mathrm{OCH}_{3}\right), 3.62\left(\mathrm{~s}, \mathrm{OCH}_{3}\right), 4.11$ (m, tert. CH), $5.20\left(\mathrm{~d}, J_{43}=7.6 \mathrm{~Hz}, 4-\mathrm{H}\right), 5.79(\mathrm{~s}, 8-\mathrm{H}), 5.81\left(\mathrm{dd}, J_{43}=7.6 \mathrm{~Hz}, J_{31}=1 \mathrm{~Hz}\right.$, $3-\mathrm{H}), 6.21\left(\mathrm{~d}, J_{6^{\prime} 2^{\prime}}=1.4 \mathrm{~Hz}, 2^{\prime}-\mathrm{H}\right), 6.36(\mathrm{~s}, 5-\mathrm{H}), 6.44\left(\mathrm{dd}, J_{6^{\prime} 5^{\prime}}=8 \mathrm{~Hz}, J_{6^{\prime} 2^{\prime}}=1.4 \mathrm{~Hz}, 6^{\prime}-\mathrm{H}\right)$, $6.60\left(\mathrm{~d}, J_{6^{\prime} 5^{\prime}}=8 \mathrm{~Hz}, 5^{\prime}-\mathrm{H}\right)$.

$$
\mathrm{C}_{21} \mathrm{H}_{25} \mathrm{NO}_{4} \text { (355.4) Ber. C } 70.94 \text { H } 7.09 \text { N } 3.94 \text { Gef. C } 70.99 \text { H } 7.10 \text { N } 3.97
$$

[1-D]-1,2-Dihydro-2-methylpapaverin ([1-D]-9): Bei der Darstellung (analog wie bei der nichtdeuterierten Verbindung) kocht man zur Vervollständigung der Umsetzung nach Zugabe des

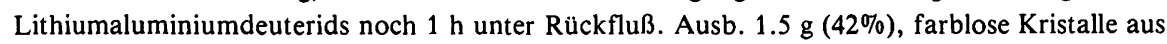
Ether, Schmp. $119.5-122.5^{\circ} \mathrm{C}$ (Zers.). - ${ }^{1} \mathrm{H}-\mathrm{NMR}$ ([D $\left.\mathrm{D}_{6}\right]$ Benzol): $\delta=2.49\left(\mathrm{~s}, \mathrm{NCH}_{3}\right), 2.91$ (s, $\left.\mathrm{CH}_{2}\right), 3.37,3.38,3.41$ und $3.45\left(\mathrm{~s}, 4 \mathrm{OCH}_{3}\right), 5.44\left(\mathrm{~d}, J_{43}=7.5 \mathrm{~Hz}, 4-\mathrm{H}\right), 5.85\left(\mathrm{~d}, J_{43}=7.5 \mathrm{~Hz}\right.$, $3-\mathrm{H}), 5.99(\mathrm{~s}, 8-\mathrm{H}), 6.39\left(\mathrm{~d}, J_{6^{\prime} 2^{\prime}}=1.4 \mathrm{~Hz}, 2^{\prime}-\mathrm{H}\right), 6.47-6.57\left(\mathrm{~m}, 5-\mathrm{H}, 5^{\prime}-\mathrm{H}\right.$ und $\left.6^{\prime}-\mathrm{H}\right)$.

3-(3,4-Dimethoxybenzyl)-3,4-dihydro-6,7-dimethoxy-2-methylisochinolinium-perchlorat (Produkt der Knabe-Umlagerung, 11)

a) direkte Darstellung: $1.9 \mathrm{~g}(5.4 \mathrm{mmol}) 9$ werden in $200 \mathrm{ml}$ Ethanol $/ 2 \mathrm{~N} \mathrm{HCl}(1: 1)$ unter Stickstoffatmosphäre gelöst und bei $25-30^{\circ} \mathrm{C}$ ca. $1.5 \mathrm{~h}$ umgelagert. Man engt die nun gelbe Lösung i. Vak. auf $100 \mathrm{ml}$ ein und gibt 40proz. Perchlorsäure zu. Der gelbe, ölige Niederschlag wird mit 
Wasser gewaschen und aus Methanol/Ether oder Methylenchlorid/Petrolether umkristallisiert.

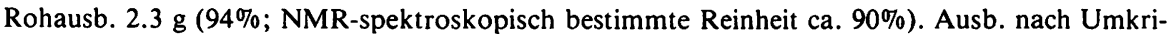
stallisation $0.4 \mathrm{~g}(16 \%)$. Schmp. $93-98^{\circ} \mathrm{C}$, Lit. ${ }^{8 a)} 176-178^{\circ} \mathrm{C}$. - IR (KBr): $2930 \mathrm{w}(\mathrm{CH})$; $1650 \mathrm{~m}, 1560 \mathrm{~m}, 1510 \mathrm{~s} \mathrm{~cm}^{-1}$. - UV (Methanol): $\lambda_{\max }(\lg \varepsilon)=249$ (4.30), $312(4.01), 368 \mathrm{~nm}$ (3.94); Lit. ${ }^{8 a):} 249$ (4.31), 311.5 (4.03), $368 \mathrm{~nm}$ (3.96). - UV (Ethylenglycolmonomethylether): $\lambda_{\max }(\lg \varepsilon)=249.5$ (4.29), 311.5 (3.98), $368 \mathrm{~nm}$ (3.93). - ${ }^{1} \mathrm{H}-\mathrm{NMR}\left(\mathrm{D}_{2} \mathrm{O} / \mathrm{DCl} / \mathrm{CD}_{3} \mathrm{OD}\right): \delta=$ 2.82 bis $3.23(\mathrm{~m}, 3 \mathrm{H}), 3.56\left(\mathrm{dd}, J_{1}=18 \mathrm{~Hz}, J_{2}=9 \mathrm{~Hz}, 1 \mathrm{H}, 4-\mathrm{H}\right) 3.78\left(\mathrm{~s}, \mathrm{OCH}_{3}\right), 3.80\left(\mathrm{~s}, \mathrm{OCH}_{3}\right)$, $3.86\left(\mathrm{~s}, \mathrm{NCH}_{3}\right), 3.99\left(\mathrm{~s}, \mathrm{OCH}_{3}\right), 4.42(\mathrm{mc}, 3-\mathrm{H}), 6.68\left(\mathrm{dd}, J_{6^{\prime} \mathrm{S}^{\prime}}=8 \mathrm{~Hz}, J_{6^{\prime} 2^{\prime}}\right.$ nicht aufgelöst, $\left.6^{\prime}-\mathrm{H}\right), 6.78\left(\mathrm{~d}, J_{6^{\prime} 2^{\prime}}\right.$ nicht aufgelöst, $\left.2^{\prime}-\mathrm{H}\right), 6.87\left(\mathrm{~d}, J_{6^{\prime} 5^{\prime}}=8 \mathrm{~Hz}, 5^{\prime}-\mathrm{H}\right), 7.04(\mathrm{~s}, 5-\mathrm{H}), 7.24(\mathrm{~s}, 8-\mathrm{H})$, $8.81(\mathrm{~s}, 1-\mathrm{H})$.

\section{$\mathrm{C}_{21} \mathrm{H}_{26} \mathrm{ClNO}_{8}$ (455.9) Ber. C 55.33 H 5.75 N 3.07 Gef. C 55.37 H 5.78 N 3.06}

b) über das „Pseudocyanid“ 148a): Die gemäß a) umgelagerte und auf $100 \mathrm{ml}$ eingeengte Reaktionslösung wird aufgearbeitet, wie in Lit. ${ }^{8 a)}$ beschrieben. 14 wird aus Petrolether/Methylenchlorid umkristallisiert. Rohausb. an $11600 \mathrm{mg}$ (88\%), nach Umkristallisation $210 \mathrm{mg}$ (31\%). Schmp. und spektroskopische Eigenschaften stimmen mit der unter a) erhaltenen Verbindung überein.

1-(3,4-Dimethoxybenzyl)-1,2,3,4-tetrahydro-6,7-dimethoxy-2-methylisochinolin (12): $300 \mathrm{mg}$ $(0.85 \mathrm{mmol}) 9$ reduziert man in $10 \mathrm{ml}$ Ethanol und $1 \mathrm{ml}$ Wasser mit $2 \mathrm{~g} \mathrm{(53} \mathrm{mmol)} \mathrm{Natriumbor-}$ hydrid. Nach üblicher Aufarbeitung ${ }^{4)}$ erhält man $290 \mathrm{mg}(95 \%)$ 12. Ausb. nach Umkristallisation aus Ether/Methanol $160 \mathrm{mg}(53 \%)$, Schmp. $114.5^{\circ} \mathrm{C}$, Lit. $.^{4)} 116-117^{\circ} \mathrm{C}$. - IR (KBr): $2930 \mathrm{~m}$, $2910 \mathrm{~m}, 2835 \mathrm{w}, 2780 \mathrm{w}(\mathrm{C}-\mathrm{H}) ; 1605 \mathrm{w}, 1585 \mathrm{w}, 1510 \mathrm{~s}$ (aromat. C - C) $\mathrm{cm}^{-1}$. - ${ }^{1} \mathrm{H}-\mathrm{NMR}$ $\left(\mathrm{CDCl}_{3}\right): \delta=2.52\left(\mathrm{~s}, \mathrm{NCH}_{3}\right), 2.37-2.91(\mathrm{~m}, 3 \mathrm{H}), 3.0-4.0(\mathrm{~m}, 4 \mathrm{H}), 3.56\left(\mathrm{~s}, \mathrm{OCH}_{3}\right), 3.81(\mathrm{~s}$, $\left.\mathrm{OCH}_{3}\right), 3.83\left(\mathrm{~s}, \mathrm{OCH}_{3}\right), 3.86\left(\mathrm{~s}, \mathrm{OCH}_{3}\right), 6.02(\mathrm{~s}, 8-\mathrm{H}), 6.52(\mathrm{~s}, 5-\mathrm{H}), 6.57\left(\mathrm{~d}, J_{6^{\prime} 2^{\prime}}=2 \mathrm{~Hz}, 2^{\prime}-\mathrm{H}\right)$, $6.62\left(\mathrm{dd}, J_{6^{\prime} 5^{\prime}}=8 \mathrm{~Hz}, J_{6^{\prime} 2^{\prime}}=2 \mathrm{~Hz}, 6^{\prime}-\mathrm{H}\right), 6.75\left(\mathrm{~d}, J_{6^{\prime} 5^{\prime}}=8 \mathrm{~Hz}, 5^{\prime}-\mathrm{H}\right) .-{ }^{1} \mathrm{H}-\mathrm{NMR}\left(\mathrm{CDCl}_{3} / \mathrm{CCl}_{4}\right)$ : $\delta=5.97(8-\mathrm{H}), 6.51(5-\mathrm{H}), 6.56\left(2^{\prime}-\mathrm{H}\right), 6.59\left(6^{\prime}-\mathrm{H}\right), 6.72\left(5^{\prime}-\mathrm{H}\right),-{ }^{13} \mathrm{C}-\mathrm{NMR}\left(\mathrm{CDCl}_{3}\right): \delta=25.83$ (C-4), $40.85\left(\right.$ Benzyl- $\left.\mathrm{CH}_{2}\right), 42.79\left(\mathrm{NCH}_{3}\right), 47.30(\mathrm{C}-3), 55.69,55.86,55.89$ und $56.02\left(\mathrm{OCH}_{3}\right)$, 64.95 (C-1), $111.43,111.46$ und 111.58 (C-5, C-8 und C-2'), $113.50\left(C^{\prime}-5^{\prime}\right.$ oder $\left.C-2^{\prime}\right), 121.99$ $\left(C-6^{\prime}\right), 126.38$ (C-4a), 129.60 (C-8a), $132.73\left(\right.$ C-1 $\left.^{\prime}\right), 146.61,147.49,147.54$ und 148.77 (C-6, C-7, C-3' und $\left.\mathrm{C}^{\prime} 4^{\prime}\right)$; Zuordnung mit Hilfe eines Spinecho-Experiments.

3-(3,4-Dimethoxybenzyl)-1,2,3,4-tetrahydro-6,7-dimethoxy-2-methylisochinolin (13): Zunächst wird 11 wie oben beschrieben durch Knabe-Umlagerung dargestellt. Die ethanolisch-salzsaure Reaktionslösung wird nach Neutralisation mit Natriumhydrogencarbonat mit Natriumborhydrid reduziert und wie üblich ${ }^{4)}$ aufgearbeitet. Ausb. $1.0 \mathrm{~g} \mathrm{(71 \% ),} \mathrm{Schmp.} 98^{\circ} \mathrm{C}$, Lit. ${ }^{4)} 100-101^{\circ} \mathrm{C}$. IR (KBr): $2940 \mathrm{~m}, 2830 \mathrm{~m}, 2755 \mathrm{~m}(\mathrm{C}-\mathrm{H}) ; 1608 \mathrm{w}, 1586 \mathrm{w}, 1512 \mathrm{~s}$ (aromat. C - C) cm ${ }^{-1}$. - ${ }^{1} \mathrm{H}-$ NMR $\left(\mathrm{CDCl}_{3}\right): \delta=2.36-2.67(\mathrm{~m}, 2 \mathrm{H}), 2.54\left(\mathrm{~s}, \mathrm{NCH}_{3}\right), 2.92-3.13(\mathrm{~m}, 2 \mathrm{H}), 3.77(\mathrm{~m}, 2 \mathrm{H})$, $3.6-4.0(\mathrm{~m}$, tert. $\mathrm{CH}-\mathrm{N}), 3.75,3.81,3.84$ und $3.86\left(\mathrm{~s}, 4 \mathrm{OCH}_{3}\right), 6.50$ und $6.55(\mathrm{~s}, 5-\mathrm{H}$ und $8-\mathrm{H})$, $6.66-6.72\left(\mathrm{~m}, 2^{\prime}-\mathrm{H}\right.$ und $\left.6^{\prime}-\mathrm{H}\right), 6.82\left(\mathrm{~d}, J_{6^{\prime} 5^{\prime}}=8 \mathrm{~Hz}, 5^{\prime}-\mathrm{H}\right) .-{ }^{13} \mathrm{C}-\mathrm{NMR}\left(\mathrm{CDCl}_{3}\right): \delta=30.93$ (C-4), 35.99 (benzyl. $\mathrm{CH}_{2}$ ), $40.86\left(\mathrm{NCH}_{3}\right), 55.37$ (C-3), $55.96\left(4 \mathrm{OCH}_{3}\right), 60.46(\mathrm{C}-1), 109.39$, $111.49,111.85$ und 112.71 (C-5, C-8, C-3' und C-5'), 121.28 (C-6 $\left.6^{\prime}\right), 125.51$ und 125.98 (C-4a und C-8a), 132.43 (C-1'), 147.46, 147.55, 147.72 und 149.01 (C-6, C-7, C-3' und C-4').

\section{6,7-Dimethoxy-2-methylisochinolinium-iodid}

a) 3,4-Dihydro-6,7-dimethoxyisochinolin 37,38): Man mischt $30 \mathrm{~g}$ (0.17 mol) 2-(3,4-Dimethoxyphenyl)ethylamin und $21 \mathrm{~g}(0.46 \mathrm{~mol})$ wasserfreie Ameisensäure und erhitzt $2.5 \mathrm{~h}$ auf $190 \mathrm{bis}$ $200^{\circ} \mathrm{C}$. Nach Lit. ${ }^{37)}$ soll hierbei 2-(3,4-Dimethoxyphenyl)- $N$-formylethylamin entstehen. Die Behandlung der Reaktionsmischung mit verd. Salzsäure und mit Natronlauge ergibt jedoch, daß sich diese Verbindung höchstens in Spuren bildet und der überwiegende Teil aus nicht umgesetztem Ausgangsmaterial besteht. Weitere Umsetzung mit $\mathrm{POCl}_{3}$ siehe Lit. ${ }^{37)}$. Rohausb. $23 \mathrm{~g}$ (73\%), 
Lit. ${ }^{38)}$ 97\%, gelbliches Öl, Reinheit ca. 90\% (NMR-spektroskopisch bestimmt). - Schmp. des Methoiodids $197^{\circ} \mathrm{C}$ (Zers.), Lit. ${ }^{39)} 202^{\circ} \mathrm{C}$, gelbes Kristallpulver (aus Methylenchlorid). - ${ }^{1} \mathrm{H}$ NMR $\left(\mathrm{CDCl}_{3}\right): \delta=2.56\left(\mathrm{t}, J=7.8 \mathrm{~Hz}\right.$, Benzyl- $\left.\mathrm{CH}_{2}\right), 3.61$ (t, verbreitert, $J=7.8 \mathrm{~Hz}$, $\left.=\mathrm{N}-\mathrm{CH}_{2}\right), 3.80\left(\mathrm{~s}, 2 \mathrm{OCH}_{3}\right), 6.51(\mathrm{~s}, 5-\mathrm{H}), 6.73(\mathrm{~s}, 8-\mathrm{H}), 8.08(\mathrm{~s}$, verbreitert, 1-H). - Aufgrund der thermischen Zersetzlichkeit wird das Rohprodukt (wie auch in Lit. ${ }^{38)}$ ) weiter umgesetzt.

b) 6,7-Dimethoxyisochinolin ${ }^{38)}: 15 \mathrm{~g}$ (ca. $70 \mathrm{mmol}$ ) rohes 3,4-Dihydro-6,7-dimethoxyisochinolin aus a) wird mit $100 \mathrm{ml}$ Decalin und portionsweise unter Schütteln mit $5 \mathrm{~g}$ Palladium (10\%) auf Aktivkohle versetzt und $1.5 \mathrm{~h}$ auf $190^{\circ} \mathrm{C}$ erhitzt.

Nach Aufarbeitung ${ }^{38)}$ wird das erhaltene Öl fraktionierend destilliert. Die Fraktionen mit Siedepunkten zwischen 130 und $150^{\circ} \mathrm{C} / 0.2$ Torr $(9.5 \mathrm{~g}$ farbloses hochviskoses Öl) werden gesammelt. Sie enthalten außer dem gewünschten 6,7-Dimethoxyisochinolin ca. 26\% 3,4-Dihydro-6,7dimethoxyisochinolin und ca. 20\% 1,2,3,4-Tetrahydro-6,7-dimethoxyisochinolin (NMR-spektroskop. Nachweis). Abweichend von der Lit.-Vorschrift löst man dieses Öl in $20 \mathrm{ml}$ Ethanol und $5 \mathrm{ml}$ Wasser und gibt 70proz. Perchlorsäure zu, bis die Mischung sauer reagiert. Der entstandene blaßgelbe Niederschlag wird in der Hitze gelöst und filtriert. Aus der langsam erkaltenden Lösung fallen blaßgelbe feine Nadeln von reinem 6,7-Dimethoxyisochinolinium-perchlorat aus. Ausb. $6.2 \mathrm{~g}\left(31 \%\right.$, bezogen auf eingesetztes 3,4-Dihydro-6,7-dimethoxyisochinolin), Schmp. $245^{\circ} \mathrm{C}$. ${ }^{1} \mathrm{H}-\mathrm{NMR}\left(\mathrm{D}_{2} \mathrm{O} /\left[\mathrm{D}_{6}\right] \mathrm{DMSO}\right): \delta=4.00$ und $4.04\left(\mathrm{~s}, \mathrm{OCH}_{3}\right), 7.55$ und $7.65(\mathrm{~s}, 5-\mathrm{H}$ und $8-\mathrm{H}), 8.10$ (d, $J=6 \mathrm{~Hz}, 4-\mathrm{H}), 8.27$ (d, $J=6 \mathrm{~Hz}, 3-\mathrm{H}), 9.20(\mathrm{~s}, 1-\mathrm{H})$.

Durch Zugabe von $2 \mathrm{~N} \mathrm{NaOH}$ und Extraktion mit Methylenchlorid $(3 \times 40 \mathrm{ml})$ erhält man nach Abdampfen des Lösungsmittels ein farbloses Öl, welches beim Stehenlassen bei $5^{\circ} \mathrm{C}$ durchkristallisiert. Ausb. $4.5 \mathrm{~g} \mathrm{(30 \% )}$, Schmp. $92^{\circ} \mathrm{C}$, Lit..$^{40)} 92^{\circ} \mathrm{C}$. - IR (KBr): $2930 \mathrm{w}(\mathrm{C}-\mathrm{H}) ; 1610 \mathrm{~m}, 1585 \mathrm{w}$, $1570 \mathrm{~m}(\mathrm{C}-\mathrm{C}) ; 1245 \mathrm{~s} \mathrm{~cm}^{-1}$. - ${ }^{1} \mathrm{H}-\mathrm{NMR}\left(\mathrm{CDCl}_{3}\right): \delta=3.97\left(\mathrm{~s}, 2 \mathrm{OCH}_{3}\right), 6.97$ und $7.12(\mathrm{~s}, 5-\mathrm{H}$ und $8-\mathrm{H}), 7.42(\mathrm{~d}, J=5.8 \mathrm{~Hz}, 4-\mathrm{H}), 8.30(\mathrm{~d}, J=5.8 \mathrm{~Hz}, 3-\mathrm{H}), 8.95(\mathrm{~s}, 1-\mathrm{H})$.

c) 6,7-Dimethoxy-2-methylisochinolinium-iodid: $4.0 \mathrm{~g}(21.2 \mathrm{mmol})$ 6,7-Dimethoxyisochinolin kocht man mit $30 \mathrm{ml}$ Methyliodid $1 \mathrm{~h}$ unter Rückfluß. Die erkaltete Mischung saugt man ab, wäscht mit Ether nach und trocknet das hellgelbe Kristallpulver i.Vak. über Phosphorpentoxid. Überschüssiges Methyliodid wird durch Destillation zurückgewonnen. Ausb. $6.5 \mathrm{~g}(93 \%)$, Schmp. $238^{\circ} \mathrm{C}$ (Zers.), Lit. ${ }^{40)} 237^{\circ} \mathrm{C}$. - IR (KBr): $3000 \mathrm{~m}, 2960 \mathrm{~m}(\mathrm{C}-\mathrm{H}) ; 1630 \mathrm{~m}, 1610 \mathrm{~m}$ $(\mathrm{C}-\mathrm{C}) ; 1275 \mathrm{~s} \mathrm{~cm}^{-1}$. - UV (Ethanol/2 $\mathrm{N} \mathrm{HCl}$ ): $\lambda_{\max }(\mathrm{lg} \varepsilon)=252$ (4.87), $310 \mathrm{~nm}$ (4.12). UV (Ethylenglycolmonomethylether): $\lambda_{\max }(\lg \varepsilon)=312 \mathrm{~nm}$ (3.78). $-{ }^{1} \mathrm{H}-\mathrm{NMR}\left(\mathrm{D}_{2} \mathrm{O} / \mathrm{CD}_{3} \mathrm{OD}\right): 4.10$ $\left(\mathrm{s}, \mathrm{OCH}_{3}\right), 4.15\left(\mathrm{~s}, \mathrm{OCH}_{3}\right), 4.47\left(\mathrm{~s}, \mathrm{NCH}_{3}\right), 7.64$ und $7.74(\mathrm{~s}, 5-\mathrm{H}$ und $8-\mathrm{H}), 8.23(\mathrm{~d}, J=6.8 \mathrm{~Hz}$, 4-H), $8.35(\mathrm{~d}, J=6.8 \mathrm{~Hz}, 3-\mathrm{H}), 9.36(\mathrm{~s}, 1-\mathrm{H})$.

1,2,3,4-Tetrahydro-6,7-dimethoxy-2-methylisochinolin ${ }^{41,42)}$ : Darstellung nach Lit. ${ }^{41)}$ aus 2-(3,4-Dimethoxyphenyl)- $N$-methylethylamin ${ }^{42}$. Das Rohprodukt wird in Abwandlung der Lit.Vorschrift ${ }^{41)}$ durch Destillation bei 0.15 Torr gereinigt. Man erhält ein farbloses Öl, das in der Vorlage kristallisiert. Ausb. $4.5 \mathrm{~g}(53 \%)$, Sdp. $119^{\circ} \mathrm{C} / 0.15$ Torr, Lit. $.^{43)} 210^{\circ} \mathrm{C} / 40$ Torr. Schmp. $78^{\circ} \mathrm{C}$, Lit. ${ }^{41)} 75-77^{\circ} \mathrm{C}$. - IR (KBr): $2930 \mathrm{~m}, 2790 \mathrm{~m}(\mathrm{C}-\mathrm{H}) ; 1605 \mathrm{~m}$ (aromat. C-C), $1515 \mathrm{~s}$ $\mathrm{cm}^{-1}$. - ${ }^{1} \mathrm{H}-\mathrm{NMR}\left(\mathrm{CDCl}_{3} / \mathrm{CCl}_{4}\right): \delta=2.41\left(\mathrm{~s}, \mathrm{NCH}_{3}\right), 2.61\left(\mathrm{t}, J=6 \mathrm{~Hz}\right.$, aromat. $\left.\mathrm{CH}_{2}\right), 2.78(\mathrm{t}$, $\left.J=6 \mathrm{~Hz}, \mathrm{~N}-\mathrm{CH}_{2}-\mathrm{CH}_{2}\right), 3.45\left(\mathrm{~s}\right.$, aromat. $\left.\mathrm{CH}_{2}-\mathrm{N}\right), 3.74\left(\mathrm{~s}, \mathrm{OCH}_{3}\right), 3.76\left(\mathrm{~s}, \mathrm{OCH}_{3}\right), 6.45$ und $6.52(\mathrm{~s}, 5-\mathrm{H}$ und $8-\mathrm{H})$.

$N$-Methylpavin $(5,6,11,12-T e t r a h y d r o-2,3,8,9$-tetramethoxy-N-methyldibenzo[a,e]cycloocten5,11-imin, 19) ${ }^{29)}$ : Darstellung nach Lit. ${ }^{29)}$ aus $450 \mathrm{mg}(1.3 \mathrm{mmol}) 9$ unter Stickstoffschutzatmosphäre. Nach Abdampfen des Lösungsmittels erhält man farblose Kristalle von N-Methylpavin. Das Produkt 11 der Knabe-Umlagerung ist UV-spektroskopisch nicht nachweisbar. Ausb. $340 \mathrm{mg}$ (76\%), Lit. ${ }^{29)} 72 \%$, aus Ether. Schmp. $140^{\circ} \mathrm{C}$, Lit. ${ }^{29)} 140-141^{\circ} \mathrm{C}$. - IR (KBr): $2890 \mathrm{~m}(\mathrm{C}-\mathrm{H})$; $1605 \mathrm{~m}(\mathrm{C}-\mathrm{C}) ; 1245 \mathrm{~s} ; 1230 \mathrm{~s} \mathrm{~cm}^{-1}$. - ${ }^{1} \mathrm{H}-\mathrm{NMR}\left(\mathrm{CDCl}_{3}\right): \delta=2.52\left(\mathrm{~s}, \mathrm{NCH}_{3}\right), 2.57\left(\mathrm{~d}, J_{1}=\right.$ 
$15.6 \mathrm{~Hz}, 2$ sekundäre $\mathrm{H}), 3.40$ (dd, $J_{1}=15.6 \mathrm{~Hz}, J_{2}=6 \mathrm{~Hz}, 2$ sekundäre $\left.\mathrm{H}\right), 3.73$ und $3.82(\mathrm{~s}$, $\left.4 \mathrm{OCH}_{3}\right), 3.79\left(\mathrm{~d}, J_{2}=6 \mathrm{~Hz}, 2\right.$ tert. $\left.\mathrm{H}\right), 6.40$ (s, 2 aromat. $\left.\mathrm{H}\right), 6.57(\mathrm{~s}, 2$ aromat. $\mathrm{H}) .-{ }^{13} \mathrm{C}-$ $\operatorname{NMR}\left(\mathrm{CDCl}_{3}\right): \delta=33.62\left(\mathrm{CH}_{2}\right), 40.92\left(\mathrm{NCH}_{3}\right), 55.74,56.01\left(4 \mathrm{OCH}_{3}\right), 56.50$ (Brückenkopf-C), $110.26,111.72$ (tert. aromat. C), 124.08, 130.17 (quart. aromat. C), 147.61, 147.98 (quart. aromat. $\mathrm{C}-\mathrm{OCH}_{3}$ ).

\section{Kinetische Messungen}

$4.70,2.79,2.35$ und $1.44 \mathrm{mg} 9$ werden jeweils in Quarzküvetten mit $1 \mathrm{~mm}$ Schichtdicke eingewogen. Man spült die Küvetten mit Stickstoff und füllt bei $20.0^{\circ} \mathrm{C}$ mit $0.325 \mathrm{ml}$ einer Mischung aus $2 \mathrm{~N} \mathrm{HCl} /$ Ethanol (1:1), welche zuvor mit einem Stickstoffstrom entgast wurde ( $c_{0}$ siehe Tab. 1). Zur Bestimmung der geeigneten Meßwellenlänge wurde die Tangentenmethode angewandt ${ }^{21)}$ : über die Wendetangente der langwelligen Flanke läßt sich bestimmen: $\lambda_{\max }=369.65 \mathrm{~nm}$, Halbwertsbreite $2 \sigma=48.8 \mathrm{~nm}$. Daraus läßt sich zusammen mit dem Extinktionskoeffizienten bei $\lambda_{\max }$ ein optimales $\varepsilon=307.4$ bei $436 \mathrm{~nm}$ berechnen. Bei dieser Wellenlänge wurde die Umlagerungskinetik jeweils kontinuierlich registriert (Auswertung siehe Tab. 1, ausführliche Fehlerrechnung siehe Lit. ${ }^{1)}$ ).

\section{${ }^{1} H$-NMR-spektroskopische Umsatzbestimmung}

Der wie oben bei 46 bzw. $21^{\circ} \mathrm{C}$ bereiteten Reaktionslösung werden in Abständen Proben entnommen und diese wie in Lit. ${ }^{4)}$ aufgearbeitet. Das erhaltene blaßgelbe Öl wird in $\mathrm{CDCl}_{3} / \mathrm{CCl}_{4}$ NMR-spektroskopisch untersucht. Aus dem Integral des Signals bei $\delta=5.97 \ldots 6.02(8-\mathrm{H}$ des hydrierten Edukts) $I_{\mathrm{E}}$ und dem Gesamtintegral der übrigen Signale zwischen 6.48 und $6.80 \mathrm{ppm}$ $I_{\mathrm{G}}$ läßt sich der Umsatz $U$ bestimmen:

$$
\text { Umsatz } U(\%)=\frac{I_{\mathrm{G}}-4 I_{\mathrm{E}}}{I_{\mathrm{G}}+I_{\mathrm{E}}} \cdot 100
$$

\section{Inhibitionsversuche}

Allgemeine Vorschrift: In einer 1:1-Mischung aus Ethanol und $2 \mathrm{~N} \mathrm{HCl}$ wird unter Stickstoffschutzatmosphäre zunächst der Inhibitor gelöst und dann unter starkem Rühren festes 9. Reaktionsparameter und Umsätze siehe Tab. 2.

1. Inhibierung durch Luftsauerstoff: $200 \mathrm{mg}(0.563 \mathrm{mmol}) 9$ versetzt man bei $21.0^{\circ} \mathrm{C}$ mit $4 \mathrm{ml}$ Ethanol $/ 2 \mathrm{~N} \mathrm{HCl}(1: 1)\left(c_{0}=0.141 \mathrm{~mol} \cdot \mathrm{1}^{-1}\right)$ und läßt $3.5 \mathrm{~h}$ an der Luft stehen. Die Reaktionslösung wird ca. $40 \mathrm{~h}$ verschlossen aufbewahrt. Man schüttelt mit $5 \mathrm{ml}$ Ether aus, trocknet die Etherphase über Molekularsieb $3 \dot{A}$ und engt an der Vigreux-Kolonne auf ca. $1 \mathrm{ml}$ ein. In diesem Rückstand werden mittels GC/MS (SE 54, 25-m-Glaskapillare, $160-200^{\circ} \mathrm{C}$ ) folgende Substanzen nachgewiesen: 3,4-Dimethoxytoluol, $m / e 153\left(16 \%, \mathrm{M}^{+}+\mathrm{H}\right), 152\left(100 \%, \mathrm{M}^{+}\right), 137(47 \%$, $\left.\mathrm{M}^{+}-\mathrm{CH}_{3}\right) ; 3,4-$ Dimethoxybenzaldehyd, $m / e 166\left(100 \%, \mathrm{M}^{+}\right), 151\left(16 \%, \mathrm{M}^{+}-\mathrm{CH}_{3}\right) ; 3,4-\mathrm{Di}-$ methoxybenzyl-ethyl-ether, $m / e 196\left(71 \%, \mathrm{M}^{+}\right), 167\left(5 \%, \mathrm{M}^{+}-\mathrm{C}_{2} \mathrm{H}_{5}\right), 151\left(100 \%, \mathrm{M}^{+}-\right.$ $\mathrm{OC}_{2} \mathrm{H}_{5}$ ).

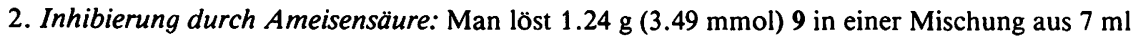
wasserfreier Ameisensäure und $1 \mathrm{ml} 2 \mathrm{~N} \mathrm{HCl}\left(c_{0}=0.436 \mathrm{~mol} \cdot 1^{-1}\right)$ und erwärmt unter Stickstoffschutzatmosphäre $4 \mathrm{~h}$ auf $85^{\circ} \mathrm{C}$. Das Lösungsmittel destilliert man im schwachen Vakuum rasch $\mathrm{ab}$, der Rückstand wird in ca. $10 \mathrm{ml}$ Wasser gelöst und $3 \mathrm{mal} \mathrm{mit} \mathrm{je} 10 \mathrm{ml}$ Ether ausgeschüttelt. Die mit Wasser gewaschenen Etherextrakte werden über Molekularsieb $3 \dot{A}$ getrocknet. Den Ether destilliert man über eine Vigreux-Kolonne bis auf ca. $0.5 \mathrm{ml} \mathrm{ab}$. Dieser Rückstand wird gaschromatographisch untersucht (DC 200, 4\%, $2 \mathrm{~m}$, gepackte Stahlsäule, $150^{\circ} \mathrm{C}$ ). Durch Zumischen von authentischer Substanz läßt sich 3,4-Dimethoxytoluol $\left(t_{\text {ret }}=8.8 \mathrm{~min}\right)$ als Nebenprodukt identifizieren. Die wäßrige Phase versetzt man mit festem Natriumhydrogencarbonat, bis keine $\mathrm{CO}_{2}$ - 
Entwicklung mehr zu beobachten ist. Man extrahiert nun $3 \mathrm{mal}$ mit Ether unter Zusatz von ca. 10\% Methylenchlorid. Nach Trocknen über Molekularsieb $3 \dot{A}$ und Abdestillieren des Lösungsmittels erhält man $800 \mathrm{mg}(64.5 \%) \mathrm{N}$-Methylpavin als farblose kristallisierte Reinsubstanz (Schmp. $140^{\circ} \mathrm{C}$, Lit. ${ }^{29)} 140-141^{\circ} \mathrm{C}$, Identifizierung durch ${ }^{1} \mathrm{H}$-NMR-Spektroskopie). In der wäßrigen Phase ist UV-spektroskopisch kein Umlagerungsprodukt nachweisbar. Man dampft diese i. Vak. zur Trockne ein und untersucht den Rückstand NMR-spektroskopisch $\left(\mathrm{CD}_{3} \mathrm{OD}\right)$. Es handelt sich um ein komplexes Substanzgemisch, bei dem die folgenden Komponenten aufgrund ihrer chemischen Verschiebung und/oder ihres Kopplungsmusters identifizierbar sind: $N$-Methylpavin (ca. 20-30\%), protoniertes Edukt der Knabe-Reaktion (10, ca. 15\%), Produkt der KnabeReaktion (11, ca.15\%; die Bildung erfolgt erst im Zuge der Aufarbeitung nach Entfernung der Ameisensäure) und 6,7-Dimethoxy-2-methylisochinoliniumsalz (ca. 5-10\%).

3. Inhibierung durch $\mathrm{DCO}_{2} \mathrm{H}: 230 \mathrm{mg}(0.647 \mathrm{mmol}) 9$ versetzt man unter $\mathrm{N}_{2}$-Schutzgas mit einer Mischung aus $1.4 \mathrm{ml} \mathrm{DCO}{ }_{2} \mathrm{H}$ (D-Gehalt ca. 99.5\%) und 3 Tropfen Ethanol/2 $\mathrm{N} \mathrm{HCl}(1: 1)$ und erwärmt 170 min auf $70^{\circ} \mathrm{C}$. Die erkaltete Reaktionsmischung verdünnt man mit $5 \mathrm{ml} \mathrm{Wasser}$ und extrahiert mit Ether $(2 \times 10 \mathrm{ml})$. Die Etherextrakte wäscht man mit Wasser und mit Kaliumcarbonatlösung, trocknet sie über Molekularsieb $3 \AA$ A und engt über eine Vigreux-Kolonne auf ca. $1 \mathrm{ml}$ ein. Eine GC-MS-Untersuchung beweist die Entstehung von nicht-deuteriertem 3,4-Dimethoxytoluol $\left[\mathrm{m} / \mathrm{e} 153\left(12 \%, \mathrm{M}^{+}+1\right), 152\left(100, \mathrm{M}^{+}\right), 137\left(54, \mathrm{M}^{+}-\mathrm{CH}_{3}\right) ; \mathrm{Zumischen}\right.$ von authentischer Probe beweist die Identität]. Die wäßrige Phase wird wie unter 2. aufgearbeitet. Es entstehen die gleichen Produkte in ähnlichen Mengen.

4. Inhibierung in $\mathrm{D}_{2} \mathrm{O} /$ Ameisensäure: Man versetzt $200 \mathrm{mg}(0.563 \mathrm{mmol}) 9$ unter $\mathrm{N}_{2}$-Schutzgas und Ausschluß von Feuchtigkeit mit einem Gemisch aus $1.2 \mathrm{ml} \mathrm{D}_{2} \mathrm{O}$ (D-Gehalt ca. 99\%), $0.1 \mathrm{ml}$ konz. Salzsäure und $0.7 \mathrm{ml}$ Ameisensäure. Man erwärmt $180 \mathrm{~min}$ auf $65^{\circ} \mathrm{C}$. Die erkaltete Reaktionsmischung wird wie unter 3. aufgearbeitet. Eine GC-MS-Untersuchung beweist die Entstehung von 3,4-Dimethoxytoluol mit einem D-Gehalt von 35.2\% [m/e 154 (9.8\%), 153 (66.1), 152 (100)]. Zweifach deuteriertes 3,4-Dimethoxytoluol entsteht im Rahmen der Meßgenauigkeit nicht.

\section{Photochemische Umsetzung von Iodbenzol mit $N, N$-Dimethyl-methylenimmonium-trifluoracetat}

Unter Feuchtigkeitsausschluß und Stickstoffschutzatmosphäre löst man $5.13 \mathrm{~g}$ (30 mmol) $N, N$ Dimethyl-methylenimmonium-trifluoracetat ${ }^{44)}$ in $200 \mathrm{ml}$ absol. Acetonitril und $30.6 \mathrm{~g}$ (150 mmol) Iodbenzol. Man bestrahlt die Lösung $20 \mathrm{~h}$ mit einer Quecksilberdampflampe ${ }^{12)}$ (Tauchlampe). Die rot gefärbte Lösung wird i. Vak. eingedampft. Den Rückstand versetzt man mit $10 \mathrm{ml}$ verd. Schwefelsäure und extrahiert mit Chloroform. Man stellt mit konz. Ammoniaklösung alkalisch und schüttelt dreimal mit je $20 \mathrm{ml}$ Chloroform aus. Die mit Wasser gewaschenen und über Magnesiumsulfat getrockneten Extrakte werden eingedampft. Ausb. $1 \mathrm{~g}(25 \%)$, gelbliches Öl, physikalische und spektroskopische Daten stimmen mit einer authentischen Probe überein.

1) Aus der Dissertation Elke Fritz-Langhals, Univ. Freiburg 1983.

2) J. Knabe, J. Kubitz und N. Ruppenthal, Angew. Chem. 75, 981 (1963).

3) J. Knabe und R. Heckmann, Arch. Pharm. (Weinheim, Ger.) 313, 1033 (1980).

4) $R$. G. Kinsman und S. F. Dyke, Tetrahedron 35, 857 (1979), und dort zitierte Literatur.

5) D. W. Brown, S. F. Dyke, R. G. Kinsman und M. Sainsbury, Tetrahedron Lett. 1969, 1731, und dort zitierte Literatur.

6) J. Knabe und K. Detering, Chem. Ber. 99, 2873 (1966).

7) E. Baldwin, S. E. Branz und J. A. Walker, J. Org. Chem. 42, 4142 (1977).

8) 8a) J. Knabe und R. Dörr, Arch. Pharm. (Weinheim, Ger.) 306, 784 (1973). - 8b) J. Knabe und $A$. Ecker, Arch. Pharm. (Weinheim, Ger.) 312, 273, 492 (1979).

9) S. z. B. K. Schwetlick, Kinetische Methoden zur Untersuchung von Reaktionsmechanismen, 1. Aufl., S. 204, VEB Deutscher Verlag der Wissenschaften, Berlin 1971. 
10) 10a) Y. L. Chow, W. C. Danen, S. F. Nelson und D. H. Rosenblatt, Chem. Rev. 78, 243 (1978). - 10b) F. Minisci, Fortschr. Chem. Forsch. 62, 1 (1976). - 10c) C. J. Michejda, D. H. Campbell, D. H. Sieh und S. R. Koepke, ACS-Symposium Series 69, 292 (1978).

11) 11a) J. M. Tedder, Angew. Chem. 94, 433 (1982); Angew. Chem., Int. Ed. Engl. 21, 401 (1982). - 11b) B. Giese, Angew. Chem. 95, 771 (1983); Angew. Chem., Int. Ed. Engl. 22, 753 (1983).

12) R. K. Sharma und N. Kharasch, Angew. Chem. 80, 69 (1968); Angew. Chem., Int. Ed. Engl. 7, 36 (1968).

13) H. Powilleit, Dissertation Univ. Saarbrücken 1969.

14) P. S. Landis in Mechanisms of Molecular Migration (B. S. Thyagarayan editor), Vol. 2, S. 43, Interscience Publ., New York 1969.

15) A. P. Kozikowski und E. Huie, J. Am. Chem. Soc. 104, 2059 (1982).

16) $M$. Meier und $C$. Rüchardt, Tetrahedron Lett., im Druck.

17) 17a) H. Schmid und B. Karrer, Helv. Chim. Acta 32, 960 (1949). - 17b) A. Pictet und B. Athanasescu, Ber. Dtsch. Chem. Ges. 33, 2346 (1900).

18) D. H. R. Barton, R. H. Hesse und G.W. Kirby, J. Chem. Soc. (London) 1965, 6379.

19) C. R. Chhabra, J. R. Kershaw und B. C. Uff, Tetrahedron Lett. 1967, 3199.

20) D. R. Dalton, M. P. Cava und K. T. Buck, Tetrahedron Lett. 1965, 2687.

21) $H$. Langhals, Ber. Bunsenges. Phys. Chem. 83, 730 (1979).

22) S. z. B. G. Pannetier und $P$. Souchan, Chemical Kinetics, 1. Aufl., S. 147, Elsevier Publ., Amsterdam 1967.

23) S. z. B. K. Schwetlick, H. Dunken, G. Pretzschner, K. Scherzer und H. J. Tiller, Chemische Kinetik, 1. Aufl., S. 119, Verlag Chemie, Weinheim 1973.

24) A. R. Forrester, J. M. Hay und R. H. Thompson, Organic Chemistry of Stable Free Radicals, 1. Aufl., S. 226, Academic Press, London 1968.

25) C. Finkentey, E. Langhals und H. Langhals, Chem. Ber. 116, 2394 (1983).

26) $M$. Anbar und $P$. Neta, J. Chem. Soc. A 1967, 837.

27) C. Walling und E. S. Huyser, Org. React. 13, 91 (1963).

28) S. z. B. 28a) F. Minisci, O. Porta und G. Sesana, J. Am. Chem. Soc. 99, $7960(1977) . ~-28 b) A$. Citterio, F. Minisci und V. Franchi, J. Org. Chem. 45, 4752 (1980). - 28c) I. Fleming, Grenzorbitale und Reaktionen organischer Verbindungen, 1. Aufl., S. 224, Verlag Chemie, Weinheim 1979.

29) A. R. Battersby und R. Binks, J. Chem. Soc. 1955, 2888.

30) M. Sainsbury, D. W. Brown, S. F. Dyke, R. G. Kinsman und B. J. Moon, Tetrahedron 24, 6695 (1968).

31) E. S. Huyser, Free Radical Chain Reactions, 1. Aufl., Wiley-Interscience 1970.

32) 32a) $M$. S. Kharasch, W. H. Urry und B. M. Kuderna, J. Org. Chem. 14, 248 (1949). 32b) S. Lit. ${ }^{31)}$ S. $25,80,152$. - 32c) R. O. C. Norman und P. R. West, J. Chem. Soc. B 1969, 389. - 32d) F. Minisci und O. Porta, Adv. Heterocycl. Chem. 16, 123 (1974).

33) Der Ort der Deuterierung ist aus der Fragmentierung im Massenspektrum nicht zu bestimmen, dennoch ist es sehr wahrscheinlich, daß es sich um [ $\alpha-D]-16$ handelt.

34) J. Knabe, W. Krause und K. Sierocks, Arch. Pharm. (Weinheim, Ger.) 303, 255 (1970).

35) J. Knabe und G. Link, Arch. Pharm. (Weinheim, Ger.) 309, 72 (1976).

36) J. Knabe, Arch. Pharm. (Weinheim, Ger.) 292, 416 (1959).

37) $R$. D. Haworth, J. Chem. Soc. (London) 1927, 2281.

38) F. D. Popp und W. E. McEwen, J. Am. Chem. Soc. 79, 3773 (1957).

39) E. Späth und H. Epstein, Ber. Dtsch. Chem. Ges. 59, 2791 (1926).

40) E. P. Taylor, J. Chem. Soc. (London) 1951, 1150.

41) J. S. Buck, J. Am. Chem. Soc. 56, 1769 (1934).

42) J. S. Buck, J. Am. Chem. Soc. 52, 4119 (1930).

43) F. L. Pyman, J. Chem. Soc. (London) 1909, 1738.

44) A. Ahond, A. Cavé, C. Kan-Fan und P. Potier, Bull. Soc. Chim. Fr. 1970, 2707.

$[221 / 83]$ 